\title{
Physics of the non-Abelian Coulomb phase: Insights from Padé approximants
}

\author{
Thomas A. Ryttov ${ }^{1}$ and Robert Shrock ${ }^{2}$ \\ ${ }^{1} C P^{3}$-Origins, University of Southern Denmark, Campusvej 55, Odense, Denmark \\ ${ }^{2}$ C. N. Yang Institute for Theoretical Physics and Department of Physics and Astronomy, \\ Stony Brook University, Stony Brook, New York 11794, USA
}

(Received 18 October 2017; published 8 January 2018)

\begin{abstract}
We consider a vectorial, asymptotically free $\mathrm{SU}\left(N_{c}\right)$ gauge theory with $N_{f}$ fermions in a representation $R$ having an infrared (IR) fixed point. We calculate and analyze Padé approximants to scheme-independent series expansions for physical quantities at this IR fixed point, including the anomalous dimension, $\gamma_{\bar{\psi} \psi}, \mathrm{IR}$, to $O\left(\Delta_{f}^{4}\right)$, and the derivative of the beta function, $\beta_{\mathrm{IR}}^{\prime}$, to $O\left(\Delta_{f}^{5}\right)$, where $\Delta_{f}$ is an $N_{f}$-dependent expansion variable. We consider the fundamental, adjoint, and rank-2 symmetric tensor representations. The results are applied to obtain further estimates of $\gamma_{\bar{\psi} \psi, \mathrm{IR}}$ and $\beta_{\mathrm{IR}}^{\prime}$ for several $\mathrm{SU}\left(N_{c}\right)$ groups and representations $R$, and comparisons are made with lattice measurements. We apply our results to obtain new estimates of the extent of the respective non-Abelian Coulomb phases in several theories. For $R=F$, the limit $N_{c} \rightarrow \infty$ and $N_{f} \rightarrow \infty$ with $N_{f} / N_{c}$ fixed is considered. We assess the accuracy of the scheme-independent series expansion of $\gamma_{\bar{\psi} \psi, \text { IR }}$ in comparison with the exactly known expression in an $\mathcal{N}=1$ supersymmetric gauge theory. It is shown that an expansion of $\gamma_{\bar{\psi} \psi, \text { IR }}$ to $O\left(\Delta_{f}^{4}\right)$ is quite accurate throughout the entire non-Abelian Coulomb phase of this supersymmetric theory.
\end{abstract}

DOI: $10.1103 /$ PhysRevD.97.025004

\section{INTRODUCTION}

The properties of a vectorial, asymptotically free gauge theory at an infrared fixed point (IRFP) of the renormalization group (RG) in a conformally invariant regime are of fundamental interest. Of equal interest is the determination of the infrared phase structure of such a theory. Owing to the asymptotic freedom, one can perform reliable perturbative calculations in the deep ultraviolet (UV) where the gauge coupling approaches zero, and then follow the renormalization-group flow to the infrared. This flow is described by the beta function, $\beta=d \alpha / d \ln \mu$, where $\alpha=g^{2} /(4 \pi), g=g(\mu)$ is the running gauge coupling, and $\mu$ is a Euclidean energy-momentum scale. For a given gauge group $G$ and fermion representation $R$ of $G$, the requirement of asymptotic freedom places an upper bound, denoted $N_{u}$, on the number of fermions, $N_{f}$, transforming according to this representation. The UV to IR evolution of a theory is determined by $G, R$, and $N_{f}$. If $N_{f}$ is slightly less than $N_{u}$, then the RG flows from the UV to a weakly coupled IRFP in a non-Abelian Coulomb phase (NACP,

Published by the American Physical Society under the terms of the Creative Commons Attribution 4.0 International license. Further distribution of this work must maintain attribution to the author(s) and the published article's title, journal citation, and DOI. Funded by SCOAP. also called the conformal window) in the infrared. The value of $\alpha$ at the IRFP is denoted $\alpha_{\mathrm{IR}}$. At this IRFP, the theory is scale-invariant and is deduced to be conformally invariant [1]. Physical quantities at this IRFP can be expressed perturbatively as series expansions in powers of $\alpha_{\mathrm{IR}}$ (e.g., [2-5]). However, beyond the lowest loop orders, the coefficients in these expansions depend on the scheme used for regularization and renormalization of the theory. Consider an asymptotically free vectorial gauge theory with gauge group $G$ and $N_{f}$ massless [6] Dirac fermions $\psi_{i}, i=1, \ldots, N_{f}$, in a representation $R$ of $G$, such that the RG flow leads to an IRFP, and let $N_{u}$ denote the value of $N_{f}$ at which asymptotic freedom is lost. Since $\alpha_{\mathrm{IR}}$ becomes small as $N_{f}$ approaches $N_{u}$ from below, one can reexpress physical quantities as series expansions in the manifestly scheme-independent variable

$$
\Delta_{f}=N_{u}-N_{f}
$$

Some early work on this was reported in $[7,8]$.

Two quantities of considerable interest are anomalous dimensions of (gauge-invariant) fermion bilinear operators and the derivative of the beta function, evaluated at the IRFP,

$$
\left.\frac{d \beta}{d \alpha}\right|_{\alpha=\alpha_{\mathrm{IR}}} \equiv \beta_{\mathrm{IR}}^{\prime} .
$$


These are physical quantities and hence are schemeindependent [9]. The derivative $\beta_{\mathrm{IR}}^{\prime}$ is equivalent to the anomalous dimension of $\operatorname{Tr}\left(F_{\mu \nu} F^{\mu \nu}\right)$, where $F_{\mu \nu}^{a}$ is the field-strength tensor of the theory [10,11]. In earlier work we had presented values of $\gamma_{\bar{\psi} \psi, \text { IR }}$ [2] (see also [3]) and $\beta_{\mathrm{IR}}^{\prime}$ [4] calculated as conventional $n$-loop series expansions in powers of the $n$-loop ( $n \ell$ ) IR coupling $\alpha_{\mathrm{IR}, n \ell}$. In [12-18] we calculated values of $\gamma_{\bar{\psi} \psi, \mathrm{IR}}$ and $\beta_{\mathrm{IR}}^{\prime}$ via our schemeindependent expansions.

In this paper we use our series expansions of $\gamma_{\bar{\psi} \psi, \text { IR }}$ to $O\left(\Delta_{f}^{4}\right)$ and of $\beta_{\mathrm{IR}}^{\prime}$ to $O\left(\Delta_{f}^{5}\right)$ at an IR fixed point in vectorial, asymptotically free gauge theories with gauge group $\mathrm{SU}\left(N_{c}\right)$ and $N_{f}$ Dirac fermions transforming according to various representations $R$, to calculate Padé approximants to these quantities. We consider $R$ equal to the fundamental $(F)$, adjoint $(A)$ and rank-2 symmetric $\left(S_{2}\right)$ tensor representations. For technical convenience, as before, we restrict our discussion to mass-independent schemes [19] and zero fermion mass [6]. We use these Padé approximants for several purposes, which also constitute motivations for this work. First, as closed-form rational functions of $\Delta_{f}$, the Padé approximants yield values of $\gamma_{\bar{\psi} \psi, \mathrm{IR}}$ and $\beta_{\mathrm{IR}}^{\prime}$ that complement the values obtained from the finite series expansions in powers of $\Delta_{f}$ and can be compared with them. These values are of fundamental importance as properties of conformal field theories and are of value in the resurgent investigation of these theories in four spacetime dimensions. Second, our calculations of these quantities in continuum quantum field theory are complementary to the program of lattice simulations to measure them, and we compare our values with lattice measurements. Third, given an asymptotically free theory with a particular choice of gauge group $G$ and fermion representation $R$, the question of what the infrared properties are, as a function of $N_{f}$, is of fundamental fieldtheoretic importance. This applies, in particular, to the question of the extent of the non-Abelian Coulomb phase as a function of $N_{f}$. Since the upper end of the NACP at $N_{u}$ [see Eq. (2.1) below] [20] is known exactly, the determination of the extent of the NACP as a function of $N_{f}$ is equivalent to the determination of the value of $N_{f}$ at the lower end of the NACP, which we denote $N_{f, c r}$. As in our earlier work, here we use our new calculations of $\gamma_{\bar{\psi} \psi \mu, \mathrm{IR}}$ using Padé approximants, together with an upper bound on this anomalous dimension from conformal invariance, to obtain further estimates of $N_{f, c r}$ and hence of the extent of the NACP for several theories. Fourth, we carry out Taylorseries expansions of our Padé approximants in powers of $\Delta_{f}$ to determine their predictions for higher-order coefficients in the scheme-independent expansions of $\gamma_{\bar{\psi} \psi, \mathrm{IR}}$ and $\beta_{\mathrm{IR}}^{\prime}$ going beyond the respective orders $O\left(\Delta_{f}^{4}\right)$ and $O\left(\Delta_{f}^{5}\right)$ to which we have calculated these. We use these to test our conjecture in earlier work that the coefficients in the series expansion of $\gamma_{\bar{\psi} \psi, \text { IR }}$ are positive. (In contrast, we have shown from our calculations for general $G$ and $R$ in [14-16] that the coefficients in the series expansions of $\beta_{\mathrm{IR}}^{\prime}$ in powers of $\Delta_{f}$ have mixed signs.) A fifth use of our Padé calculations pertains to phenomenology. In addition to the importance of $N_{f, c r}$ as a basic property describing the UV to IR evolution and infrared phase structure of an asymptotically free gauge theory, this is also important for phenomenological studies, since a knowledge of $N_{f, c r}$ is crucial for the construction of quasiconformal gauge theories as possible candidates for ultraviolet completions of the Standard Model. This is because, for a given $G$ and $R$, these constructions of quasiconformal theories require that one choose $N_{f}$ to be slightly less than $N_{f, c r}$ in order to achieve the quasiconformal behavior whose spontaneous breaking via chiral symmetry breaking could have the potential to yield a light, dilatonic Higgs-like scalar. As a sixth part of the present work, we present a new analytic result concerning the accuracy of the finite series expansion of $\gamma_{\bar{\psi} \mu, \mathrm{IR}}$ in powers of $\Delta_{f}$ as compared with the exactly known result in an $\mathcal{N}=1$ supersymmetric gauge theory. This analytical result extends our earlier demonstrations $[12,15-17]$ that the truncation of the series for $\gamma_{\bar{\psi} \psi, \text { IR }}$ to $O\left(\Delta_{f}^{4}\right)$ is quite accurate throughout the entire non-Abelian Coulomb phase of this supersymmetric theory.

This paper is organized as follows. In Sec. II we briefly review relevant background and methodology. In Secs. III and IV we present our results for $\gamma_{\bar{\psi} \mu, \text { IR }}$ and $\beta_{\mathrm{IR}}^{\prime}$, respectively. We give our conclusions in Sec. V.

\section{BACKGROUND AND METHODS}

\section{A. General}

Here we briefly review some background and methods relevant for our work. We refer the reader to our previous papers [12-18] for details. The requirement of asymptotic freedom implies that $N_{f}$ must be less than an upper $(u)$ bound $N_{u}$, where [20]

$$
N_{u}=\frac{11 C_{A}}{4 T_{f}} .
$$

Here, $C_{2}(R)$ is the quadratic Casimir invariant for the representation $R, C_{A}=C_{2}(A)$, where $A$ is the adjoint representation, and $T_{f} \equiv T(R)$ is the trace invariant [21].

At the maximal scheme-independent loop order, namely the two-loop level, the beta function has an IR zero if $N_{f}$ lies in the interval $I$ defined by

$$
I: N_{\ell}<N_{f}<N_{u}
$$

where $N_{u}$ was given in Eq. (2.1) and 


$$
N_{\ell}=\frac{17 C_{A}^{2}}{4 T_{f}\left(5 C_{A}+3 C_{f}\right)}
$$

The maximum value of $\Delta_{f}$ for $N_{f} \in I$ is

$$
\Delta_{f, \max } \equiv N_{u}-N_{\ell}=\frac{3 C_{A}\left(6 C_{A}+11 C_{f}\right)}{4 T_{f}\left(5 C_{A}+3 C_{f}\right)} .
$$

Formally generalizing $N_{f}$ from positive integers $\mathbb{N}_{+}$to positive real numbers, $\mathbb{R}_{+}$, one can let $N_{f}$ approach $N_{u}$ from below, thereby making $\alpha_{\mathrm{IR}}$ arbitrarily small. Thus, for the UV to IR evolution in this regime of $N_{f}$, one infers that the theory evolves from weak coupling in the UV to an IRFP in a non-Abelian Coulomb phase. As stated above, we denote the lowest value of $N_{f}$ in this NACP as $N_{f, c r}$, and correspondingly, we define [22]

$$
\Delta_{f, c r} \equiv N_{u}-N_{f, c r}
$$

So

$$
\mathrm{NACP}: N_{f, c r}<N_{f}<N_{u} \text {. }
$$

One of the goals of lattice studies of these types of gauge theories has been to estimate $N_{f, c r}$ for a given $G$ and $R$ [23]. We have also obtained estimates of $N_{f, c r}$ by combining our $O\left(\Delta_{f}^{4}\right)$ calculations of $\gamma_{\bar{\psi} \psi, \text { IR }}$ with our finding of monotonicity and the conformality upper bound [24] $\gamma_{\bar{\psi} \psi, \text { IR }} \leq 2$ in our earlier work [13-16]. We will discuss this further below in connection with our new Padé calculations. Our computations assume that the IRFP is exact, as is the case in the non-Abelian Coulomb phase [25]. In the analytic expressions and plots given below, this restriction, that $N_{f}$ lies in the NACP, will be understood implicitly. In Table I we tabulate some relevant values of $N_{\ell}, N_{u}, \Delta_{f \text {,max }}$, and the intervals with $N_{f} \in \mathbb{R}_{+}$and $N_{f} \in \mathbb{N}_{+}$.

For the gauge group $G=\mathrm{SU}\left(N_{c}\right)$ and the specific fermion representations $R$ considered in this paper, the general formulas above for $N_{\ell}, N_{u}$, and $\Delta_{f \text { max }}$ read as follows:

$$
\begin{aligned}
& R=F: \quad N_{\ell}=\frac{34 N_{c}^{3}}{13 N_{c}^{2}-3}, \quad N_{u}=\frac{11 N_{c}}{2}, \\
& \Delta_{f, \max }=\frac{3 N_{c}\left(25 N_{c}^{2}-11\right)}{2\left(13 N_{c}^{2}-3\right)}, \\
& R=A: \quad N_{\ell}=\frac{17}{16}=1.0625, \quad N_{u}=\frac{11}{4}=2.75, \\
& R=S_{2}: \quad \Delta_{\ell, \max }=\frac{27}{16}=1.687 N_{c}^{3} \\
&\left(N_{c}+2\right)\left(8 N_{c}^{2}+3 N_{c}-6\right) \\
& N_{u}=\frac{11 N_{c}}{2\left(N_{c}+2\right)}, \\
& \Delta_{f, \max }=\frac{3 N_{c}\left(18 N_{c}^{2}+11 N_{c}-22\right)}{2\left(N_{c}+2\right)\left(8 N_{c}^{2}+3 N_{c}-6\right)} .
\end{aligned}
$$

We list these values for the theories under consideration in Table I.

\section{B. Scheme-independent expansion for $\gamma_{\bar{\psi} \mu \text {.IR }}$}

Since the global chiral symmetry is realized exactly in the non-Abelian Coulomb phase, the bilinear fermion operators can be classified according to their representation properties under this symmetry, including flavor-singlet and flavor-nonsinglet. The anomalous dimensions evaluated at the IRFP are the same for these flavor-singlet and flavor-nonsinglet fermion bilinears [26]. For $R=F$, these are $\quad \bar{\psi} \psi \equiv \sum_{i=1}^{N_{f}} \bar{\psi}_{i} \psi_{i}$ and $\bar{\psi} \mathcal{T}_{b} \psi \equiv \sum_{i, j=1}^{N_{f}} \bar{\psi}_{i}\left[\mathcal{T}_{b}\right]_{i j} \psi_{j}$, where $\mathcal{T}_{b}$ is a generator of $\mathrm{SU}\left(N_{f}\right)$, and similarly for other representations. Hence, as in our earlier work, we use the symbol $\gamma_{\bar{\psi} \mu, \text { IR }}$ to refer to both. This anomalous dimension at the IRFP has the scheme-independent expansion

$$
\gamma_{\bar{\psi} \mu, \mathrm{IR}}=\sum_{j=1}^{\infty} \kappa_{j} \Delta_{f}{ }^{j} .
$$

We denote the truncation of the infinite series in Eq. (2.10) at $j=s$ as $\gamma_{\bar{\psi} \psi, \mathrm{IR}, \Delta_{f}^{s}}$. When it is necessary for clarity, we indicate the representation $R$ explicitly in the subscripts as $\gamma_{\bar{\psi} \psi, I R, R}, \gamma_{\bar{\psi} \mu} \mu, \mathrm{IR}, R, \Delta_{f}^{s}$, and $\kappa_{j, R}$. In general, the calculation of

TABLE I. $\quad N_{\ell}, N_{u}, \Delta_{f, \max }$, and interval $I$ in terms of $N_{f}$, for $G=\mathrm{SU}\left(N_{c}\right)$ with fermions in the representation $R$ equal to fundamental $(\mathrm{F})$, adjoint $(\mathrm{A})$, and rank-2 symmetric $\left(\mathrm{S}_{2}\right)$ tensor. The interval $I$ is listed for $N_{f}$ formally generalized to real numbers, $\mathbb{R}_{+}$and for physical, integral values of $N_{f} \in \mathbb{N}_{+}$. Note that for $R=A, N_{\ell}$ and $N_{u}$ are independent of $N_{c}$.

\begin{tabular}{llccccc}
\hline \hline$N_{c}$ & $R$ & $N_{\ell}$ & $N_{u}$ & $\Delta_{f, \max }$ & $I, N_{f} \in \mathbb{R}_{+}$ & $I, N_{f} \in \mathbb{N}_{+}$ \\
\hline 2 & $\mathrm{~F}$ & 5.551 & 11 & 5.449 & $5.551<N_{f}<11$ & $6 \leq N_{f} \leq 10$ \\
3 & $\mathrm{~F}$ & 8.053 & 16.5 & 8.447 & $8.053<N_{f}<16.5$ & $9 \leq N_{f} \leq 16$ \\
4 & $\mathrm{~F}$ & 10.615 & 22 & 11.385 & $10.615<N_{f}<22$ & $11 \leq N_{f} \leq 21$ \\
$N_{c}$ & $\mathrm{~A}$ & 1.0625 & 2.75 & 1.6875 & $1.0625<N_{f}<1.6875$ & $N_{f}=2$ \\
3 & $\mathrm{~S}_{2}$ & 1.224 & 3.3000 & 2.076 & $1.224<N_{f}<3.300$ & $N_{f}=2,3$ \\
4 & $\mathrm{~S}_{2}$ & 1.353 & 3.3667 & 2.313 & $1.353<N_{f}<3.667$ & $N_{f}=2,3$ \\
\hline \hline
\end{tabular}


the coefficient $\kappa_{j}$ requires, as inputs, the $\ell$-loop coefficients in the conventional loop expansion of the beta function in powers of $\alpha$, namely $b_{\ell}$, with $1 \leq \ell \leq j+1$, and the $\ell$-loop coefficients $c_{\ell}$ in the corresponding conventional expansion of $\gamma_{\bar{\psi} \psi \psi}$ with $1 \leq \ell \leq j$. The coefficients $\kappa_{j}$ were calculated for general gauge group $G$ and fermion representation $R$ for $1 \leq j \leq 3$ in [12] and for $j=4$ in [16]. The calculation of $\kappa_{4}$ was given for $\mathrm{SU}(3)$ and $R=F$ in [13] and for $\mathrm{SU}\left(N_{c}\right)$ in [15], Our calculation of $\kappa_{4}$ for general $G$ and $R$ used the $b_{\ell}$ coefficients up to $b_{5}$ from [27] and $c_{\ell}$ up to $c_{4}$ from [28]. Specific expressions for $\kappa_{j}$ and plots of $\gamma_{\bar{\psi} \psi \mu, \mathrm{IR}, \Delta_{f}^{s}}$ for $G=\mathrm{SU}\left(N_{c}\right)$ and the fundamental, adjoint, and rank-2 symmetric and antisymmetric representations were given in [12-17] and for $G=\mathrm{SO}\left(N_{c}\right)$ and $\mathrm{Sp}\left(N_{c}\right)$ in [18]. In [14] we discussed operational criteria for the accuracy of the $\Delta_{f}$ expansion, and we briefly review some points here.

As with usual perturbative series expansions in powers of interaction couplings in quantum field theories, the $\Delta_{f}$ expansion is generically an asymptotic expansion rather than a Taylor series. This follows from the fact that in order for an expansion in a variable $z$ to be a Taylor series with finite radius of convergence, it is necessary (and sufficient) that the function for which the series is calculated must be analytic at the origin of the complex $z$ plane. In particular, with $z=\Delta_{f}$, this means that the properties of the theory should not change qualitatively as one moves from real positive $\Delta_{f}$ through the point $\Delta_{f}=0$ to negative $\Delta_{f}$, i.e., as $N_{f}$ increases through the value $N_{u}$. However, one knows that the properties of the theory do change qualitatively as $N_{f}$ increases beyond $N_{u}$, namely it ceases to be asymptotically free. Nevertheless, as with conventional perturbative expansions in powers of the interaction coupling in quantum field theory, one may get a rough estimate of the accuracy of a truncated series by performing the ratio test on the series coefficients that have been calculated. This type of procedure is used, for example, in perturbative quantum electrodynamics and quantum chromodynamics calculations in powers of the respective interaction couplings, and we gave results on the relevant ratios of terms in our series expansions of $\gamma_{\bar{\psi} \psi, \mathrm{IR}}$ and $\beta_{\mathrm{IR}}^{\prime}$ in our previous work [12-16]. These, in conjunction with plots of curves, gave quantitative evaluations of the accuracy of the $\Delta_{f}$ expansions for these quantities. We will expand upon this earlier work here by comparing $O\left(\Delta_{f}^{s}\right)$ expansions for $\gamma_{\bar{\psi} \psi, \text { IR }}$ with the exactly known expression for this anomalous dimension in an $\mathcal{N}=$ 1 supersymmetric gauge theory below.

Let us denote the full scaling dimension of an operator $\mathcal{O}$ as $D_{\mathcal{O}}$ and its free-field value as $D_{\mathcal{O} \text {,free }}$. We define the anomalous dimension of $\mathcal{O}$, denoted $\gamma_{\mathcal{O}}$, by

$$
D_{\mathcal{O}}=D_{\mathcal{O}, \text { free }}-\gamma_{\mathcal{O}}
$$

Given that the theory at an IRFP in the non-Abelian phase is conformally invariant, there is a conformality lower bound on $D_{\mathcal{O}}$ from unitarity, namely $D_{\bar{\psi} \psi \psi} \geq 1$ [24]. Since $D_{\bar{\psi} \mu \text {,free }}=3$, this is equivalent to the upper bound

$$
\gamma_{\bar{\psi} \psi, \mathrm{IR}} \leq 2 .
$$

In [13], for $\mathrm{SU}(3)$ and $R=F$, using our calculation of $\gamma_{\bar{\psi} \psi, \mathrm{IR}}$ to $O\left(\Delta_{f}^{4}\right)$, i.e., $\gamma_{\bar{\psi} \psi}, \mathrm{IR}, \Delta_{f}^{4}$, we used polynomial extrapolation to obtain estimates of the evaluation of the infinite series (2.10) yielding the value of $\gamma_{\bar{\psi} \psi, \text { IR }}$ as a function of $N_{f}$. We compared our results with lattice measurements for $N_{f}=12$ and $N_{f}=10$.

In the series expansion (2.10) for $\gamma_{\bar{\psi} \psi, \text { IR }}$, the first two coefficients, $\kappa_{1}$ and $\kappa_{2}$, are manifestly positive for any gauge group $G$ and fermion representation $R$ [12]. Although $\kappa_{3}$ and $\kappa_{4}$ contain terms with negative as well as positive signs, one of the important results of our explicit calculations of $\kappa_{3}$ and $\kappa_{4}$ for $\mathrm{SU}\left(N_{c}\right), \mathrm{SO}\left(N_{c}\right)$, and $\mathrm{Sp}\left(N_{c}\right)$ gauge groups and a variety of representations, including fundamental, adjoint, and rank-2 symmetric and antisymmetric tensors, was that for all of these theories, $\kappa_{3}$ and $\kappa_{4}$ are also positive [14-16,18]. Moreover, as reviewed below, in a gauge theory with $\mathcal{N}=1$ supersymmetry, an exact expression is known for the anomalous dimension of the (gauge-invariant) fermion bilinear operator, and the Taylorseries expansion of this exact expression in powers of $\Delta_{f}$ yields $\kappa_{j}$ coefficients that are all positive. These results led to our conjecture in [13], elaborated upon in our later works, that, in addition to the manifestly positive $\kappa_{1}$ and $\kappa_{2}$, and our findings in $[14-16,18]$ that $\kappa_{3}$ and $\kappa_{4}$ are positive for all of the groups and representations for which we calculated them, (i) the higher-order $\kappa_{j}$ coefficients with $j \geq 5$ are also positive in (vectorial, asymptotically free) nonsupersymmetric gauge theories. In turn, this conjecture led to several monotonicity conjectures, namely that (ii) for fixed $s, \gamma_{\bar{\psi} \psi, \mathrm{IR}, \Delta_{f}^{s}}$ increases monotonically as $N_{f}$ decreases in the non-Abelian Coulomb phase, and (iii) for fixed $N_{f}$ in the NACP, $\gamma_{\bar{\psi} \psi, I R, \Delta_{f}^{s}}$ is a monotonically increasing function of $s$, so that (iv) for fixed $N_{f}$ in the NACP and for finite $s$, $\gamma_{\bar{\psi} \psi, \mathrm{IR}, \Delta_{f}^{s}}$ is a lower bound on the actual anomalous dimension $\gamma_{\bar{\psi} \psi, \mathrm{IR}}$, as defined by the infinite series (2.10). By similar reasoning, the analogous conjectures apply for the $k$ th derivatives of the anomalous dimension as a function of $\Delta_{f}$. In particular, for the first derivative, one has the analogous conjectures $(\text { ii })_{d}$ for fixed $s$,

$$
\frac{d\left[\gamma_{\bar{\psi} \mu, \mathrm{IR}, \Delta_{f}^{s}}\right]}{d \Delta_{f}}=-\frac{d\left[\gamma_{\bar{\psi} \mu, \mathrm{IR}, \Delta_{f}^{s}}\right]}{d N_{f}}
$$

increases monotonically as $\Delta_{f}$ increases, i.e., as $N_{f}$ decreases, in the NACP [where the subscript $d$ on (ii) $d$ connotes "derivative"]; $(\text { iii })_{d}$ for fixed $N_{f}$ in the NACP, $\frac{d}{d \Delta_{f}}\left[\gamma_{\bar{\psi} \psi, \mathrm{IR}, \Delta_{f}^{s}}\right]$ is a monotonically increasing function of $s$, so that (iv) ${ }_{d}$ for fixed $N_{f}$ in the NACP and for finite $s$, the 
derivative $\frac{d}{d \Delta_{f}}\left[\gamma_{\bar{\psi} \psi}, \mathrm{IR}, \Delta_{f}^{s}\right]$ is a lower bound on the derivative $\frac{d}{d \Delta_{f}}\left[\gamma_{\bar{\psi} \psi, \mathrm{IR}}\right]$ of the actual anomalous dimension, as defined by the infinite series (2.10).

Combining our calculations of $\gamma_{\bar{\psi} \psi, \text { IR }}$ to $O\left(\Delta_{f}^{4}\right)$ with these positivity and resultant monotonicity conjectures (used as assumptions), and with the further assumption that $\gamma_{\bar{\psi} \mu, \text { IR }}$ saturates its conformality upper bound of 2 in (2.12), as $N_{f}$ decreases to $N_{f, c r}$ at the lower end of the nonAbelian Coulomb phase, we have then derived estimates of $N_{f, c r}$ in various theories [29]. For example, in [13] we inferred that $N_{f, c r}=8-9[13,29]$. In $[15,16]$ we extended these $O\left(\Delta_{f}^{4}\right)$ calculations of $\gamma_{\bar{\psi} \psi, \text { IR }}$ from the special case of $\mathrm{SU}(3)$ and $R=F$ to general $G$ and $R$, and, for $G=\mathrm{SU}\left(N_{c}\right)$ with various $N_{c}$ and $R$, we again compared our values of $\gamma_{\bar{\psi} \mu, \mathrm{IR}, \Delta_{f}^{4}}$ with values from lattice measurements.

Here, we extend this program further via the calculation and evaluation of Padé approximants for $G=\mathrm{SU}\left(N_{c}\right)$ with several values of $N_{c}$ and several fermion representations $R$. There are a number of applications of these calculations: (i) to get further estimates of the value of the anomalous dimension of the fermion bilinear for various $N_{c}$ and fermion representations $R$; (ii) to estimate $N_{f, c r}$, as just described; and (iii) via Taylor series expansions of the Padé approximants, to determine their predictions for higherorder coefficients $\kappa_{j}$ with $j \geq 5$. Of course, regarding application (iii), since the Padé approximants that we calculate for $\gamma_{\bar{\psi} \psi, \text { IR }}$ are based on the series (2.10) computed only up to $O\left(\Delta_{f}^{4}\right)$, their predictions for these higher-order $\kappa_{j}$ with $j \geq 5$ only provide a hint as to their actual values.

\section{Scheme-independent expansion for $\beta_{\mathrm{IR}}^{\prime}$}

Given the property of asymptotic freedom, $\beta$ is negative in the region $0<\alpha<\alpha_{\mathrm{IR}}$, and since $\beta$ is continuous, it follows that this function passes through zero at $\alpha=\alpha_{\mathrm{IR}}$ with positive slope, i.e., $\beta_{\mathrm{IR}}^{\prime}>0$. This derivative $\beta_{\mathrm{IR}}^{\prime}$ has the scheme-invariant expansion

$$
\beta_{\mathrm{IR}}^{\prime}=\sum_{j=2}^{\infty} d_{j} \Delta_{f}^{j} .
$$

As indicated, $\beta_{\mathrm{IR}}^{\prime}$ has no term linear in $\Delta_{f}$. In general, the calculation of the scheme-independent coefficient $d_{j}$ requires, as inputs, the $\ell$-loop coefficients in the beta function, $b_{\ell}$, for $1 \leq \ell \leq j$. We denote the truncation of the infinite series in Eq. (2.14) at $j=s$ as $\beta_{\mathrm{IR}, \Delta_{f}^{s}}^{\prime}$.

Let the full scaling dimension of $\operatorname{Tr}\left(F_{\mu \nu} F^{\mu \nu}\right)$ be denoted $D_{F_{\mu \nu}^{2}}$, with free-field value 4 . (With operator mixing, this refers to the matrix element in the anomalous dimension matrix.) At an IRFP, $D_{F^{2}, \mathrm{IR}}=4+\beta_{\mathrm{IR}}^{\prime}[10,11]$, so $\beta_{\mathrm{IR}}^{\prime}=-\gamma_{F^{2}}$,IR. Given that the theory at an IRFP in the non-Abelian phase is conformally invariant, there is a conformality bound from unitarity, namely $D_{F^{2}} \geq 1$ [24]. Since $\beta_{\mathrm{IR}}^{\prime}>0$, this bound is obviously satisfied.

As part of our work, we will calculate Padé approximants to our series expansions to $O\left(\Delta_{f}^{5}\right)$ for $\beta_{\mathrm{IR}}^{\prime}$. We will use these for the analogues of the applications (i) and (iii) mentioned above for $\gamma_{\bar{\psi} \psi, \text { IR }}$, namely to obtain additional information about the value of $\beta_{\mathrm{IR}}^{\prime}$ and to get some hints regarding coefficients $d_{j}$ going beyond the order to which we have calculated them, i.e., with $j \geq 6$.

\section{LNN limit}

For $G=\mathrm{SU}\left(N_{c}\right)$ and $R=F$, it is of interest to consider the limit

$$
\begin{array}{ll}
L N N: & N_{c} \rightarrow \infty, \quad N_{f} \rightarrow \infty \\
\text { with } \quad r \equiv \frac{N_{f}}{N_{c}} \quad \text { fixed and finite } \\
\text { and } \quad \xi(\mu) \equiv \alpha(\mu) N_{c} \text { is a finite function of } \mu .
\end{array}
$$

We will use the symbol $\lim _{L N N}$ for this limit, where "LNN" stands for "large $N_{c}$ and $N_{f}$ " with the constraints in Eq. (2.15) imposed. This is also called the 't HooftVeneziano limit.

Here we give some background for our calculation of Padé approximants in the LNN limit. We define

$$
r_{u}=\lim _{L N N} \frac{N_{u}}{N_{c}},
$$

and

$$
r_{\ell}=\lim _{L N N} \frac{N_{\ell}}{N_{c}}
$$

with values

$$
r_{u}=\frac{11}{2}=5.5
$$

and

$$
r_{\ell}=\frac{34}{13}=2.615
$$

(to the indicated floating-point accuracy). With the interval $I$ defined as in Eq. (2.2), it follows that the corresponding interval in the ratio $r$ is

$$
I_{r}: \frac{34}{13}<r<\frac{11}{2}, \quad \text { i.e., } 2.615<r<5.5 \text {. }
$$

The critical value of $r$ such that for $r>r_{c r}$, the LNN theory is in the NACP and is IR-conformal, while for $r<r_{c r}$, it exhibits spontaneous chiral symmetry breaking, is denoted $r_{c r}$ and is defined as

$$
r_{c r}=\lim _{L N N} \frac{N_{f, c r}}{N_{c}} .
$$


We define the rescaled scheme-independent expansion parameter for the LNN limit

$$
\Delta_{r} \equiv \lim _{L N N} \frac{\Delta_{f}}{N_{c}}=r_{u}-r=\frac{11}{2}-r .
$$

As $r$ decreases from $r_{u}$ to $r_{\ell}$ in the interval $I_{r}, \Delta_{r}$ increases from 0 to a maximal value

$$
\Delta_{r, \max }=r_{u}-r_{\ell}=\frac{75}{26}=2.8846 \quad \text { for } r \in I_{r} .
$$

Further, we define the maximum value of $\Delta_{r}$ in the NACP as

$$
\Delta_{r, c r}=r_{u}-r_{c r}=\frac{11}{2}-r_{c r}
$$

Since $\kappa_{j} \propto N_{c}^{-j}$, the rescaled coefficients $\hat{\kappa}_{j, F}$ that are finite in the LNN limit are

$$
\hat{\kappa}_{j, F} \equiv \lim _{N_{c} \rightarrow \infty} N_{c}^{j} \kappa_{j, F}
$$

The anomalous dimension $\gamma_{\mathrm{IR}}$ is also finite in this limit and is given by

$$
R=F: \lim _{L N N} \gamma_{\mathrm{IR}}=\sum_{j=1}^{\infty} \kappa_{j, F} \Delta_{f}^{j}=\sum_{j=1}^{\infty} \hat{\kappa}_{j, F} \Delta_{r}^{j} .
$$

The appropriately rescaled beta function that is finite in the LNN limit is

$$
\beta_{\xi}=\frac{d \xi}{d t}=\lim _{L N N} N_{c} \beta
$$

where $\xi$ was defined in Eq. (2.15). Since the derivative $d \beta_{\xi} / d \xi$ satisfies the relation

$$
\frac{d \beta_{\xi}}{d \xi}=\frac{d \beta}{d \alpha} \equiv \beta^{\prime},
$$

it follows that $\beta^{\prime}$ is finite in the LNN limit (2.15). We define the rescaled coefficient

$$
\hat{d}_{j, F}=\lim _{L N N} N_{c}^{j} d_{j, F},
$$

which is finite in the LNN limit. Thus, writing $\lim _{L N N} \beta_{\mathrm{IR}}^{\prime}$ as $\beta_{\mathrm{IR}, L N N}^{\prime}$ for this $R=F$ case, we have

$$
\beta_{\mathrm{IR}, L N N}^{\prime}=\sum_{j=1}^{\infty} d_{j, F} \Delta_{f}^{j}=\sum_{j=1}^{\infty} \hat{d}_{j, F} \Delta_{r}^{j} .
$$

We denote the value of $\beta_{\mathrm{IR}, L N N}^{\prime}$ obtained from this series calculated to order $O\left(\Delta_{f}^{p}\right)$ as $\beta_{\mathrm{IR}, L N N, \Delta_{f}^{p}}^{\prime}$.

\section{E. Test of accuracy of scheme-independent expansion for $\gamma_{\bar{\psi} \psi, \text { IR }}$ using supersymmetric gauge theory}

A basic question for the scheme-independent series expansions of physical quantities at an IRFP in powers of $\Delta_{f}$ in the non-Abelian Coulomb phase is how accurate a finite truncation of this series is. We have addressed this question in previous work [12,14-16] by investigating how accurate the truncated, finite-order expansion is, as a function of $N_{f}$, in a theory where an exact expression for the anomalous dimension of the fermion bilinear is known.

Here we briefly review this analysis and give some new quantitative measures of the accuracy. For this accuracy check, we use a vectorial, asymptotically free gauge theory with $\mathcal{N}=1$ supersymmetry ( $s s$ ), gauge group $G$, and $N_{f}$ pairs of chiral superfields $\Phi_{j}$ and $\tilde{\Phi}_{j}, j=1, \ldots, N_{f}$, that transform according to the respective representations $R$ and $\bar{R}$ of $G$. The requirement of asymptotic freedom in this theory requires that $N_{f}$ must be less than an upper limit, which we will again denote $N_{u}$, namely

$$
\text { ss: } \quad N_{u}=\frac{3 C_{A}}{2 T_{f}} \text {. }
$$

(Throughout this subsection, to avoid cumbersome notation, we will use the same notation $N_{u}, N_{\ell}, N_{f, c r}$, etc. as in the nonsupersymmetric case, but it will be understood implicitly that these quantities refer to this supersymmetric theory.) In this theory, the lower end of the non-Abelian Coulomb phase occurs at $N_{f, c r}=N_{u} / 2$, so the NACP occupies the range

$$
\begin{array}{ll}
\text { ss: NACP: } \quad & \frac{N_{u}}{2}<N_{f}<N_{u}, \text { i.e., } \\
\frac{3 C_{A}}{4 T_{f}}<N_{f} & <\frac{3 C_{A}}{2 T_{f}} .
\end{array}
$$

Thus, in this theory, $\Delta_{f}$ increases from 0 to a maximum value

$$
\text { ss: } \quad \Delta_{f, \max }=\frac{N_{u}}{2}=\frac{3 C_{A}}{4 T_{f}}
$$

as $N_{f}$ decreases from $N_{u}$ at the upper end of the NACP to $N_{u} / 2$ at the lower end of the NACP.

The anomalous dimension of the quadratic chiral superfield operator product $\tilde{\Phi} \Phi$, and hence also the fermion bilinear contained in this product, are exactly known in such theories. Defining the mesonic operator $M \equiv \bar{\psi} \psi$, with a sum over group indices understood, one has [30-32] 


$$
\begin{aligned}
\gamma_{M, \mathrm{IR}} & =\frac{\Delta_{f}}{N_{u}-\Delta_{f}}=\frac{\frac{\Delta_{f}}{N_{u}}}{1-\frac{\Delta_{f}}{N_{u}}} \\
& =\sum_{j=1}^{\infty}\left(\frac{\Delta_{f}}{N_{u}}\right)^{j}
\end{aligned}
$$

This anomalous dimension $\gamma_{M \text {,IR }}$ increases monotonically from 0 at $N_{f}=N_{u}$ at the upper end of the NACP to saturate its upper limit of 1 when $N_{f}$ reaches $N_{f, c r}=N_{u} / 2$ at the lower end of the NACP. From this exact expression (2.34), it follows that the coefficient $\kappa_{j}$ in Eq. (2.10) is

$$
s s: \quad \kappa_{j}=\frac{1}{\left(N_{u}\right)^{j}}=\left(\frac{2 T_{f}}{3 C_{A}}\right)^{j} .
$$

Thus, $\kappa_{j}$ is positive for all $j$, which provided motivation for our conjecture in $[13,14]$ that $\kappa_{j}>0 \forall j$ in the nonsupersymmetric theory, in accord with the manifestly positive $\kappa_{1}$ and $\kappa_{2}$, and the positivity of the $\kappa_{j}$ with $j=3,4$ that we had calculated for each group and representation that we considered [12-16,18]. From this positivity of the $\kappa_{j}$ calculated to the highest order, $j=4$, the monotonicity property of $\gamma_{\bar{\psi} \psi, \mathrm{IR}, \Delta_{f}^{4}}$ follows. That is, our calculation of $\gamma_{\bar{\psi} \psi, \mathrm{IR}, \Delta_{f}^{4}}$ in the nonsupersymmetric theory shares with the exact expression in the $\mathcal{N}=1$ supersymmetric theory the property that it increases monotonically with decreasing $N_{f}$ in the NACP. Note that this monotonicity does not hold for the scheme-dependent conventional $n$-loop ( $n \ell$ ) calculation of $\gamma_{\mathrm{IR}, n \ell}$; for example, it was found [2,3] that for various specific theories, such as (nonsupersymmetric) $\mathrm{SU}\left(N_{c}\right)$ with $N_{c}=2,3,4$ and $R=F$, although $\kappa_{3}$ is positive in the NACP, $\kappa_{4}$ (calculated in the widely used MS scheme) is negative, so that, as $N_{f}$ decreases in the NACP, $\gamma_{\bar{\psi} \psi, \mathrm{IR}, 4 \ell}$ reaches a maximum and then decreases. In the $\mathcal{N}=1$ supersymmetric theory, we also showed that $\kappa_{3}$ (calculated in the $\overline{\mathrm{DR}}$ scheme) is negative, and, as a consequence, the scheme-dependent three-loop calculation of $\gamma_{M, I R, 3 e}$ fails to exhibit the known monotonicity of the exact result [33]. This again demonstrates the advantage of the scheme-independent series expansion at the IRFP, (2.10), in powers of $\Delta_{f}$, as compared with conventional expansions in powers of the coupling $\alpha_{\mathrm{IR}}$.

The series expansion for $\gamma_{M \text {,IR }}$ is particularly simple in the $\mathcal{N}=1$ supersymmetric gauge theory, since it is a geometric series. Because the fermions appear together with the Grassmann variable $\theta$ in the chiral superfield $\Phi_{j}=\phi_{j}+\sqrt{2} \theta \psi_{j}+\theta \theta F_{j}$ (where $F_{j}$ is an auxiliary field), the conformality lower bound $D_{\Phi} \geq 1$ on the full scaling dimension of the chiral superfield is equivalent to the conformality upper bound

$$
\gamma_{M, I R} \leq 1
$$

on the (gauge-invariant) fermion bilinear in $\Phi_{j} \tilde{\Phi}_{j}$. This upper bound is saturated as $N_{f}$ decreases to $N_{f, c r}$. Thus, as we have observed before $[12,14,16,17]$, both of the assumptions that we make for our estimate of $N_{f, c r}$ in nonsupersymmetric theories, namely that (i) $\kappa_{j}>0$ for all $j$, and (ii) $\gamma_{\bar{\psi} \psi, \text { IR }}$ saturates its upper bound from conformal invariance as $N_{f}$ decreases to the lower end of the nonAbelian Coulomb phase, are satisfied in a gauge theory with $\mathcal{N}=1$ supersymmetry. (As discussed above, the upper bounds themselves are different, namely 2 in the nonsupersymmetric theory, Eq. (2.12) and 1 in the supersymmetric theory, Eq. (2.36) [34].)

We next determine the accuracy of a finite truncation of the series (2.10). To do this we calculate the fractional difference

$$
\epsilon_{s s} \equiv \frac{\gamma_{M, \mathrm{IR}}-\gamma_{M, \mathrm{IR}, \Delta_{f}^{s}}}{\gamma_{M, \mathrm{IR}}}
$$

where we denote the finite series (2.10) for $\gamma_{M, I R}$ truncated to maximal power $j=s$ as $\gamma_{M, I R, \Delta_{f}^{s}}$. Using the elementary identity $\sum_{j=1}^{s} x^{j}=x\left(x^{s}-1\right) /(x-1)$ to sum the finite series $\sum_{j=1}^{s} \kappa_{j} \Delta_{f}^{j}$, we obtain

$$
\gamma_{M, \mathrm{IR}, \Delta_{f}^{s}}=\frac{\left(\frac{\Delta_{f}}{N_{u}}\right)\left[\left(\frac{\Delta_{f}}{N_{u}}\right)^{s}-1\right]}{\frac{\Delta_{f}}{N_{u}}-1} .
$$

Substituting this into Eq. (2.37), we find

$$
\epsilon_{s s}=\left(\frac{\Delta_{f}}{N_{u}}\right)^{s} .
$$

Since the maximum value of the ratio $\Delta_{f} / N_{u}$ is $1 / 2$, this fractional difference decreases toward zero exponentially rapidly with $s$. Quantitatively, if one sets $N_{f}=N_{u} / 2$, the value at the bottom of the NACP, then $\epsilon_{s s}=(1 / 2)^{s}=$ $e^{-(\ln 2) s}$, so the fractional difference between the $O\left(\Delta_{f}^{4}\right)$ result, $\gamma_{M, \mathrm{IR}, \Delta_{f}^{4}}$, and the exact result, $\gamma_{M, \mathrm{IR}}$, is $6.25 \%$. If one formally sets $N_{f}=(3 / 4) N_{u}$, further up in the NACP, then $\Delta_{f}=N_{u} / 4$ and the fractional difference between $\gamma_{M, \mathrm{IR}, \Delta_{f}^{s}}$ and the exact result is $\epsilon_{s s}=(1 / 4)^{s}$. This takes on the value $0.391 \%$ for $s=4$. In general, if we require that $\epsilon_{s s}<\epsilon_{0}$ for some $\epsilon_{0}>0$, then this implies that it is necessary to calculate the finite, truncated series in powers of $\Delta_{f}$ up to and including the power

$$
s=\frac{\ln \left(\frac{1}{\epsilon_{0}}\right)}{\ln \left(\frac{N_{u}}{\Delta_{f}}\right)},
$$

to achieve this fractional accuracy, where it is understood that if $s$ is a nonintegral real number, then one sets $s$ equal 
to the closest integer greater than the value in Eq. (2.40). At the upper end of the non-Abelian Coulomb phase, since $\Delta_{f} / N_{u}$ is small, $\ln \left(N_{u} / \Delta_{f}\right)$ is large and one can achieve a small fractional difference $\epsilon_{0}$ with a modest value of $s$. The most stringent requirement on $s$ to achieve a given fractional accuracy $\epsilon_{0}$ occurs as $N_{f}$ approaches the lower end of the NACP at $N_{f}=N_{u} / 2$ and is

$$
s=\frac{\ln \left(\frac{1}{\epsilon_{0}}\right)}{\ln 2} .
$$

As we have calculated, if one wants to achieve a fractional difference that is less than or equal to $6.25 \%$ for all $N_{f}$ in the NACP, then Eq. (2.40) shows that the expansion to $O\left(\Delta_{f}^{4}\right)$ is sufficient for this accuracy.

These results show quantitatively that finite truncations of the infinite series (2.10), even up to only modest maximal powers such as $s=4$, yield very accurate approximations to the exactly known anomalous dimension $\gamma_{M, \text { IR }}$ in this $\mathcal{N}=1$ supersymmetric gauge theory. In passing, we remark that if an exact expression for $\beta_{\mathrm{IR}}^{\prime}$ were available in this theory, then we could also use it to obtain an additional measure of how accurate a finite-order truncation of the infinite series (2.14) is to the exact function. However, to our knowledge, an exact expression for $\beta_{\mathrm{IR}}^{\prime}$ is not known for this theory.

This $\mathcal{N}=1$ supersymmetric gauge theory also provides a framework in which to investigate how accurate a $[p, q]$ Padé approximant to a finite-order truncation of the infinite series in Eq. (2.34) would be to the exact result in (2.34) for $\gamma_{M, I R}$. In general, if one calculates a $[p, q]$ Padé approximant for a finite truncation of such a simple series as the geometric series in (2.34), then not only does the $[0,1]$ approximant reproduce the exact function (2.34), but so do all of the $[p, q]$ approximants with $q \neq 0$. The way that they do this is by inserting factors in the numerator and denominator to yield polynomials of degree $p$ and $q$, but which cancel precisely, yielding the exact function (2.34) itself.

From the exact expression (2.34), one can also calculate the value of the derivative $d \gamma_{M, \mathrm{IR}} / d N_{f}$, which is

$$
\frac{d \gamma_{M, \mathrm{IR}}}{d N_{f}}=-\frac{d \gamma_{M, \mathrm{IR}}}{d \Delta_{f}}=-\frac{N_{u}}{N_{f}^{2}}
$$

This derivative is always negative in the NACP and increases monotonically in magnitude with decreasing $N_{f}$. It has the value $-1 / N_{u}$ for $N_{f}=N_{u}$ at the upper end of the NACP, and $-4 / N_{u}$ for $N_{f}=N_{u} / 2$ at the lower end of the NACP. The curvature is

$$
\frac{d^{2} \gamma_{M, \mathrm{IR}}}{d N_{f}^{2}}=\frac{d^{2} \gamma_{M, \mathrm{IR}}}{d \Delta_{f}^{2}}=\frac{2 N_{u}}{N_{f}^{3}} .
$$

This curvature is positive in the NACP and increases from $2 / N_{u}^{2}$ at the upper end, to $16 / N_{u}^{2}$, at the lower end, of the NACP. Given that we have shown that $\kappa_{1}$ and $\kappa_{2}$ are manifestly positive and $\kappa_{j}$ are positive for all the $G$ and $R$ for which we have evaluated them, it follows that our $\gamma_{\bar{\psi} \psi, \mathrm{IR}, \Delta_{f}^{4}}$ also has positive curvature for $N_{f}$ in the NACP, a property that it shares with the exactly known $\gamma_{M \text {,IR }}$ in the $\mathcal{N}=1$ supersymmetric theory. We showed in [33] that the three-loop calculation of $\gamma_{M, \mathrm{IR}, 3 \ell}$ in the supersymmetric gauge theory, carried out as a conventional schemedependent series expansion in powers of $\alpha$, fails to exhibit the known positive curvature of the exact result, just as it fails to exhibit the known monotonicity of the exact result. This is another advantage of the scheme-independent expansion in powers of $\Delta_{f}$.

We have noted above that the value of $N_{f}$ at the lower end of the NACP does not, in general, coincide with the value $N_{\ell}$ at the lower end of the interval $I$ where the twoloop beta function has an IR zero. For this $\mathcal{N}=1$ supersymmetric theory, one can calculate this difference exactly $[33,35]$. One has

$$
N_{\ell}=\frac{3 C_{A}^{2}}{2 T_{f}\left(C_{A}+2 C_{f}\right)} .
$$

Hence, this difference for this theory is

$$
N_{\ell}-N_{f, c r}=\frac{3 C_{A}\left(C_{A}-2 C_{f}\right)}{4 T_{f}\left(C_{A}+2 C_{f}\right)}
$$

[where here and in the rest of this subsection, $N_{\ell}$ is given by (2.44) and should not be confused with Eq. (2.3)]. This difference can be positive or negative. For example, for $G=\mathrm{SU}\left(N_{c}\right)$ and $R=F$, this difference is the positive quantity

$$
R=F: \quad N_{\ell}-N_{f, c r}=\frac{3 N_{c}}{2\left(2 N_{c}^{2}-1\right)} .
$$

This decreases to zero as $N_{c} \rightarrow \infty$. The resultant fractional difference between these values is

$$
R=F: \quad \frac{N_{\ell}-N_{f, c r}}{N_{\ell}}=\frac{1}{2 N_{c}^{2}-1} .
$$

This fractional difference (2.47) has the values 0.143 and 0.0588 for $N_{c}=2$ and $N_{c}=3$, respectively, and also decreases toward zero with increasing $N_{c}$. In contrast, for both the adjoint and symmetric rank-2 tensor representations, the difference $N_{\ell}-N_{f, c r}$ is negative and does not vanish as $N_{c} \rightarrow \infty$ (with $N_{f}$ fixed).

In the LNN limit of the $\mathcal{N}=1$ supersymmetric gauge theory with $G=\mathrm{SU}\left(N_{c}\right)$ and $R=F$, 


$$
L N N: \quad r_{c r}=r_{\ell}=\frac{3}{2},
$$

so that

$$
L N N: \quad \Delta_{r, c r}=\Delta_{r, \max }=\frac{3}{2} .
$$

\section{PADÉ APPROXIMANTS FOR $\gamma_{\bar{\psi} \mu, \text { IR }}$ USING THE $O\left(\Delta_{f}^{4}\right)$ SERIES}

\section{A. General}

In this section we report our calculation of Padé approximants to our scheme-independent $O\left(\Delta_{f}^{4}\right)$ series for $\gamma_{\bar{\psi} \psi, \mathrm{IR}}$, in $\mathrm{SU}\left(N_{c}\right)$ theories with various fermion representations $R$. (For a review of Padé approximants, see, e.g., [36].) It may be recalled that resummation methods such as Padé approximants have been useful in in the analysis of series expansions in both quantum field theories and in statistical mechanics [37] (e.g. [38-43]), and our current work extends to higher order our earlier calculations of Padé approximants for these types of gauge theories $[2,5,13,14]$.

In general, given the series calculated to maximal order $s$, denoted $\gamma_{\bar{\psi} \mu, \mathrm{IR}, \Delta_{f}^{s}}$ as above (with $N_{c}$ and $R$ implicit in the notation), we write this as

$\gamma_{\bar{\psi} \mu, \mathrm{IR}, \Delta_{f}^{s}}=\sum_{j=1}^{s} \kappa_{j} \Delta_{f}^{j}=\kappa_{1} \Delta_{f}\left[1+\frac{1}{\kappa_{1}} \sum_{j=2}^{s} \kappa_{j} \Delta_{f}^{j-1}\right]$

and calculate the $[p, q]$ Padé approximant to the expression in square brackets, with $p+q=s-1$. This takes the form

$$
\gamma_{\bar{\psi} \psi, \mathbb{I R},[p, q]}=\kappa_{1} \Delta_{f}\left[\frac{1+\sum_{i=1}^{p} \mathcal{N}_{i} \Delta_{f}^{i}}{1+\sum_{j=1}^{q} \mathcal{D}_{j} \Delta_{f}^{j}}\right],
$$

where

$$
\kappa_{1}=\frac{8 C_{f} T_{f}}{C_{A}\left(7 C_{A}+11 C_{f}\right)} .
$$

The $[p, q]$ Padé approximant is thus a rational function whose $p+q$ coefficients are determined uniquely by the condition that the Taylor series expansion of this approximant must match the $s-1$ coefficients in the series in square brackets. With the prefactor $\kappa_{1} \Delta_{f}$ thus extracted, the Padé approximant in the square brackets is normalized to be equal to 1 at $\Delta_{f}=0$. By construction, the series expansion of each closed-form approximant $\gamma_{\bar{\psi} \psi, \mathrm{IR},[p, q]}$ exactly reproduces the series expansion of $\gamma_{\bar{\psi} \psi \mu, \mathrm{IR}}$ up to the maximal order to which we have calculated it, $s=4$. In addition to providing a closed-form rational-function approximation to the finite series, a Padé approximant also can be used in another way, namely to yield a hint of higher-order terms. This information is obtained by carrying this Taylor series expansion of $[p, q]$ Padé approximants with $q \neq 0$ to higher order. We will use the Padé approximants for both of these applications.

Note that the $[s-1,0]$ Padé approximant is just the series itself, i.e.,

$$
\gamma_{\bar{\psi} \psi, \mathrm{IR},[s-1,0]}=\gamma_{\bar{\psi} \psi, \mathrm{IR}, \Delta_{f}^{s}} .
$$

As a special case of Eq. (3.4) for $j_{\max }=4, \gamma_{\bar{\psi} \psi, I R,[3,0]}$ is just the original polynomial $\gamma_{\bar{\psi} \psi, \text { IR, } \Delta_{f}^{4}}$ itself, and hence we do not consider it, since we have already obtained evaluations of this truncated series in previous work.

By construction, the $[p, q]$ Padé approximant in (3.2) is analytic at $\Delta_{f}=0$, and if it has $q \neq 0$, then it is a meromorphic function with $q$ poles. A necessary condition that must be satisfied for a Padé approximant to be useful for our analysis here is that it must not have a pole for any $\Delta_{f}$ in the interval $0<\Delta_{f}<\Delta_{c r}$, or equivalently, for any $N_{f}$ in the non-Abelian Coulomb phase. Since $N_{f, c r}$ is not precisely known for all values of $N_{c}$ and all fermion representations $R$ under consideration here, we will also use another condition, namely that the Padé approximant should not have any poles for $N_{f}$ in the interval $I$ where the two-loop beta function has an IR zero, or equivalently, for $\Delta_{f}$ in the interval $0<\Delta_{f}<\Delta_{f \text {,max }}$. This second condition can be applied in a straightforward manner for each $N_{c}$ and $R$, since the upper and lower ends of this interval $I$, namely $N_{u}$ and $N_{\ell}$, and thus $\Delta_{f, \max }$, are known [listed above in Eqs. (2.1), (2.3), and (2.4)]. Since the $[p, q]$ Padé approximant in Eq. (3.2) is an analytic function at $\Delta_{f}=0$, and, for $q \neq 0$, is meromorphic, the radius of convergence of its Taylor series expansion is set by the magnitude of the pole closest to the origin in the complex $\Delta_{f}$ plane. Let us denote this radius of convergence as $\Delta_{f \text {,conv. }}$. We shall also require that $\Delta_{f \text {,conv. }}$ be greater than $\Delta_{f, \max }$ and $\Delta_{f, c r}$, since we would like the Taylor series expansion of (3.2) to accurately reproduce the series (2.10) in this disk. We will do this to be as careful as possible, even though the actual expansion (2.10) is, in general, only expected to be an asymptotic expansion.

$$
\text { B. } \boldsymbol{G}=\mathrm{SU}\left(\boldsymbol{N}_{\boldsymbol{c}}\right), \boldsymbol{R}=\boldsymbol{F}
$$

With $G=\mathrm{SU}\left(N_{c}\right)$ and $R=F$, the explicit expression for $\kappa_{1, F}$ is

$$
\kappa_{1, F}=\frac{4\left(N_{c}^{2}-1\right)}{N_{c}\left(25 N_{c}^{2}-1\right)} .
$$

The explicit numerical expressions for the schemeindependent series expansions of $\gamma_{\bar{\psi} \mu, \mathrm{IR}}$ to order $\Delta_{f}^{4}$ for $R=F$ and $N_{c}=2,3,4$ are as follows: 


$$
\begin{aligned}
& \mathrm{SU}(2): \gamma_{\bar{\psi} \psi, \mathrm{IR}, F, \Delta_{f}^{4}}=0.0674157 \Delta_{f}+\left(0.733082 \times 10^{-2}\right) \Delta_{f}^{2}+\left(0.605308 \times 10^{-3}\right) \Delta_{f}^{3}+\left(1.626624 \times 10^{-4}\right) \Delta_{f}^{4}, \\
& \mathrm{SU}(3): \gamma_{\bar{\psi} \mu, \mathrm{IR}, F, \Delta_{f}^{4}}=0.0498442 \Delta_{f}+\left(3.79282 \times 10^{-3}\right) \Delta_{f}^{2}+\left(2.37475 \times 10^{-4}\right) \Delta_{f}^{3}+\left(3.67893 \times 10^{-5}\right) \Delta_{f}^{4}, \\
& \mathrm{SU}(4): \gamma_{\bar{\psi} \mu, \mathrm{IR}, F, \Delta_{f}^{4}}=0.0385604 \Delta_{f}+\left(2.231420 \times 10^{-3}\right) \Delta_{f}^{2}+\left(1.122984 \times 10^{-4}\right) \Delta_{f}^{3}+\left(1.265054 \times 10^{-5}\right) \Delta_{f}^{4} .
\end{aligned}
$$

In these equations,

$$
\Delta_{f}=\frac{11 N_{c}}{2}-N_{f} \quad \text { for } R=F .
$$

For reasons of space, in Eqs. (3.6)-(3.8) we list the expansions to the given floating-point accuracy; our actual algebraic computer calculations of Padé approximants use the coefficients in these expansions to considerably higher numerical accuracy.

\section{C. $\mathrm{SU}(2)$}

For $G=\mathrm{SU}(2)$ with $R=F$, the general formulas (2.1) and (2.3) give $N_{u}=11$ and $N_{\ell}=5.551$, so the interval $I$ with $N_{f} \in \mathbb{R}_{+}$is $5.551<N_{f}<11$, with $\Delta_{f, \max }=N_{u}-N_{\ell}=$ 5.449 , and the physical interval with $N_{f} \in \mathbb{N}_{+}$is $6 \leq N_{f} \leq 10$. This information is summarized in Table I. For this $\mathrm{SU}(2)$ theory with $R=F$, we calculate the following $[p, q]$ Padé approximants with $q \neq 0$ :

$$
\begin{aligned}
& \gamma_{\bar{\psi} \mu, F,[2,1]}=0.0674157 \Delta_{f}\left[\frac{1-0.159986 \Delta_{f}-0.0202427 \Delta_{f}^{2}}{1-0.268727 \Delta_{f}}\right], \\
& \gamma_{\bar{\psi} \psi, F,[1,2]}=0.0674157 \Delta_{f}\left[\frac{1+0.613518 \Delta_{f}}{1+0.504778 \Delta_{f}-0.0638685 \Delta_{f}^{2}}\right], \\
& \gamma_{\bar{\psi} \psi(F,[0,3]}=0.0674157 \Delta_{f}\left[\frac{1}{1-0.1087405 \Delta_{f}+\left(2.845756 \times 10^{-3}\right) \Delta_{f}-\left(1.745923 \times 10^{-3}\right) \Delta_{f}^{3}}\right] .
\end{aligned}
$$

The [2,1] Padé approximant in $\gamma_{\bar{\psi} \psi, F,[2,1]}$ has a pole at $\Delta_{f}=3.721$ i.e., at $N_{f}=7.279$. This is in the interval $I$ and in the estimated NACP, so we cannot use this [2,1] Padé approximant for our analysis. The [1,2] Padé approximant in $\gamma_{\bar{\psi} \psi, F,[1,2]}$ has poles at $\Delta_{f}=-1.6405$, i.e., $N_{f}=12.6405$, and at $\Delta_{f}=9.544$, i.e., $N_{f}=1.456$. The first of these occurs at a value of $N_{f}$ greater than $N_{u}$, while the second occurs at a value of $N_{f}$ well below $N_{\ell}$ and $N_{f, c r}$, so neither is in the interval $I$ or in the NACP. Finally, the [0,3] Padé approximant has a pole at $\Delta_{f}=6.289$, i.e., at $N_{f}=4.731$, which is below $N_{\ell}$ and slightly below the estimated $N_{f, c r}$. In addition, this $[0,3]$ approximant has a complexconjugate pair of poles at $\Delta_{f}=-2.3195 \pm 9.273 i$, whose magnitude is 9.558 , considerably greater than $\Delta_{f, \max }=$ 5.449 and $\Delta_{c r} \simeq 5.5$. Hence, we can use the $[1,2]$ and $[0,3]$ Padé approximants for our analysis. In Table II we list values of $\gamma_{\bar{\psi} \psi, \mathrm{IR}, F,[1,2]}$ and $\gamma_{\bar{\psi} \psi, \mathrm{IR}, F,[0,3]}$ for this $\mathrm{SU}(2)$ theory with $R=F$ as a function of $N_{f}$ and, for comparison, values of $\gamma_{\bar{\psi} \psi, \mathrm{IR}, F, \Delta_{f}^{s}}$ for $1 \leq s \leq 4$ from [14,16]. In Fig. 1 we plot these values.

We next make some general comments about these $\mathrm{SU}(2), R=F$ calculations, which also will apply to our calculations for $\mathrm{SU}(3)$ and $\mathrm{SU}(4)$ with $R=F$. In earlier work [12-18], we have noted that, in addition to the manifestly positive $\kappa_{1}$ and $\kappa_{2}$, the higher-order coefficients $\kappa_{j}$ with $j=3$ and $j=4$ are positive for all of the groups, $\mathrm{SU}\left(N_{c}\right), \mathrm{SO}\left(N_{c}\right)$, and $\operatorname{Sp}\left(N_{c}\right)$ and for all of the representations, namely $F, A, S_{2}$, and $A_{2}$, for which we have performed these calculations. Here (at an IR fixed point in the non-Abelian Coulomb phase), this means that, at least with $s$ in the range $1 \leq s \leq 4$, (i) for fixed $s, \gamma_{\bar{\psi} \psi, \mathrm{IR}, F, \Delta_{f}^{s}}$ monotonically increases with decreasing $N_{f}$; and (ii) for fixed $N_{f}$ and hence $\Delta_{f}, \gamma_{\bar{\psi} \psi, \mathrm{IR}, F, \Delta_{f}^{s}}$ is a monotonically increasing function of $s$. As is evident from Table II and Fig. 1, the analogue of the monotonicity property (i) is also true for the Padé approximants, namely that both the [1,2] and $[0,3]$ Padé approximants increase monotonically with decreasing $N_{f}$ values listed in the table and shown in the figure. The value of the anomalous dimension obtained via the $[1,2]$ Padé approximant, $\gamma_{\bar{\psi} \mu, I R, F,[1,2]}$, is quite close to $\gamma_{\bar{\psi} \psi, \mathrm{IR}, F, \Delta_{f}^{4}}$, increasing slightly above it as $N_{f}$ decreases toward the lower part of the non-Abelian Coulomb phase. The curve for $\gamma_{\bar{\psi} \psi, \mathrm{IR}, F,[0,3]}$ lies above that for $\gamma_{\bar{\psi} \psi, \mathrm{IR}, F,[1,2]}$ and increases more rapidly with decreasing $N_{f}$. Corresponding results will be given below in Table III for the LNN limit.

In $[15,16]$ we compared our results for $\gamma_{\bar{\psi} \psi, \mathrm{IR}, F, \Delta_{f}^{s}}$ with $s$ up to 4 in this $\mathrm{SU}(2)$ theory with our earlier conventional $n$-loop calculations in [2] and with lattice measurements for 
TABLE II. Values of $\gamma_{\bar{\psi} \psi, \text { IR, }, F,[1,2]}$ and $\gamma_{\bar{\psi} \psi, \mathrm{IR}, F,[0,3]}$, as functions of $N_{f}$ for $G=\mathrm{SU}\left(N_{c}\right)$ with $2 \leq N_{c} \leq 4$ and fermion representation and $R=F$. For comparison, we include values of $\gamma_{\bar{\psi} \psi, I R, F, \Delta_{f}^{s}}$ with $1 \leq s \leq 4$. Values of anomalous dimensions that exceed the conformality upper bound $\gamma_{\bar{\psi} \psi}, \mathrm{IR}=2$ are marked with brackets. To save space, we omit the $\bar{\psi} \psi$ from the subscripts in the table, writing $\gamma_{\bar{\psi} \psi, \mathrm{IR}, F, \Delta_{f}^{s}} \equiv \gamma_{\mathrm{IR}, F, \Delta_{f}^{s}}$, etc.

\begin{tabular}{lrllllll}
\hline \hline$N_{c}$ & $N_{f}$ & $\gamma_{\mathrm{IR}, F, \Delta_{f}}$ & $\gamma_{\mathrm{IR}, F, \Delta_{f}^{2}}$ & $\gamma_{\mathrm{IR}, F, \Delta_{f}^{3}}$ & $\gamma_{\mathrm{IR}, F, \Delta_{f}^{4}}$ & $\gamma_{\mathrm{IR}, F,[1,2]}$ & $\gamma_{\mathrm{IR}, F,[0,3]}$ \\
\hline 2 & 6 & 0.337 & 0.520 & 0.596 & 0.698 & 0.711 & 1.090 \\
2 & 7 & 0.270 & 0.387 & 0.426 & 0.467 & 0.466 & 0.541 \\
2 & 8 & 0.202 & 0.268 & 0.285 & 0.298 & 0.296 & 0.310 \\
2 & 9 & 0.135 & 0.164 & 0.169 & 0.172 & 0.171 & 0.173 \\
2 & 10 & 0.0674 & 0.0747 & 0.07535 & 0.0755 & 0.0755 & 0.0755 \\
3 & 8 & 0.424 & 0.698 & 0.844 & 1.036 & 1.149 & {$[2.848]$} \\
3 & 9 & 0.374 & 0.587 & 0.687 & 0.804 & 0.844 & 1.2645 \\
3 & 10 & 0.324 & 0.484 & 0.549 & 0.615 & 0.627 & 0.764 \\
3 & 11 & 0.274 & 0.389 & 0.428 & 0.462 & 0.464 & 0.5105 \\
3 & 12 & 0.224 & 0.301 & 0.323 & 0.338 & 0.3375 & 0.352 \\
3 & 13 & 0.174 & 0.221 & 0.231 & 0.237 & 0.236 & 0.240 \\
3 & 14 & 0.125 & 0.148 & 0.152 & 0.153 & 0.153 & 0.154 \\
3 & 15 & 0.0748 & 0.0833 & 0.0841 & 0.0843 & 0.0843 & 0.0843 \\
3 & 16 & 0.0249 & 0.0259 & 0.0259 & 0.0259 & 0.0259 & 0.0259 \\
4 & 11 & 0.424 & 0.694 & 0.844 & 1.029 & 1.138 & {$[2.491]$} \\
4 & 12 & 0.386 & 0.609 & 0.721 & 0.8475 & 0.897 & 1.376 \\
4 & 13 & 0.347 & 0.528 & 0.610 & 0.693 & 0.713 & 0.914 \\
4 & 14 & 0.308 & 0.451 & 0.509 & 0.561 & 0.568 & 0.656 \\
4 & 15 & 0.270 & 0.379 & 0.418 & 0.448 & 0.450 & 0.488 \\
4 & 16 & 0.231 & 0.312 & 0.336 & 0.352 & 0.352 & 0.368 \\
4 & 17 & 0.193 & 0.249 & 0.263 & 0.2705 & 0.270 & 0.276 \\
4 & 18 & 0.154 & 0.190 & 0.197 & 0.200 & 0.200 & 0.202 \\
4 & 19 & 0.116 & 0.136 & 0.139 & 0.140 & 0.140 & 0.140 \\
4 & 20 & 0.0771 & 0.0860 & 0.0869 & 0.0871 & 0.0871 & 0.0872 \\
4 & 21 & 0.0386 & 0.0408 & 0.0409 & 0.0409 & 0.0409 & 0.0409 \\
\hline \hline
\end{tabular}

$N_{f}=8[44,45]$. These lattice measurements are consistent with the $\mathrm{SU}(2), R=F, N_{f}=8$ theory being IR-conformal, so our calculations in the non-Abelian Coulomb phase are applicable. As listed in Table II, rounding off to two significant figures, we have $\gamma_{\bar{\psi} \psi, \mathrm{IR}, F, \Delta_{f}^{3}}=0.29$ and $\gamma_{\bar{\psi} \psi, \mathrm{IR}, F, \Delta_{f}^{4}}=0.30$, in very good agreement with our two Padé values, $\gamma_{\bar{\psi} \psi, \mathrm{IR}, F,[1,2]}=0.30$ and $\gamma_{\bar{\psi} \psi, \mathrm{IR}, F,[0,3]}=0.31$ and slightly higher than our earlier $n$-loop calculations $\gamma_{\bar{\psi} \psi, \mathrm{IR}, F, 3 \ell}=0.27$ and $\gamma_{\bar{\psi} \psi, \mathrm{IR}, F, 4 \ell}=0.20$.

We now go further to combine our scheme-independent series calculation of $\gamma_{\bar{\psi} \psi, \mathrm{IR}, \Delta_{f}^{4}}$ with our Padé approximant computation to estimate $N_{f, c r}$ for this theory. We require that both of the two Padé-based values, namely $\gamma_{\bar{\psi}} \psi, \mathrm{IR}, F,[1,2]$ and $\gamma_{\bar{\psi} \psi, I R, F,[0,3]}$, should obey the conformality upper bound (2.12). We also assume that the larger of these values saturates this upper bound at the lower end of the NACP, as the exactly known $\gamma_{M \text {,IR }}$ saturates its upper bound in the supersymmetric gauge theory discussed above. Then the $N_{f}$ value at which the larger of these two values exceeds

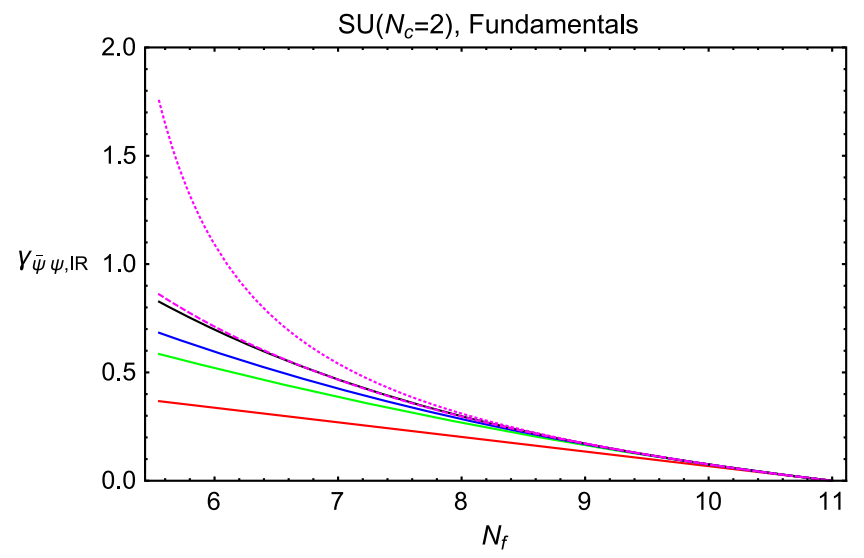

FIG. 1. Plot of $\gamma_{\bar{\psi} \psi, \mathrm{IR}, F,[1,2]}$ and $\gamma_{\bar{\psi} \psi, \mathrm{IR}, F,[0,3]}$ for $N_{c}=2$, i.e., $\mathrm{SU}(2)$ and $R=F$, together with $\gamma_{\bar{\psi} \psi, \mathrm{IR}, F, \Delta_{f}^{s}}$ with $1 \leq s \leq 4$, as a function of $N_{f}$. The vertical axis is labelled generically as $\gamma_{\bar{\psi} \psi, \mathrm{IR}}$. From bottom to top, the curves (with colors online) refer to $\gamma_{\bar{\psi} \psi, \mathrm{IR}, F, \Delta_{f}}$ (red), $\gamma_{\bar{\psi} \psi, \mathrm{IR}, F, \Delta_{f}^{2}} \quad$ (green), $\gamma_{\bar{\psi} \mu \psi, \mathrm{IR}, F, \Delta_{f}^{3}}$ (blue), and $\gamma_{\bar{\psi} \psi, \operatorname{IR}, F, \Delta_{f}^{4}}$ (black). The curves for $\gamma_{\bar{\psi} \psi \psi, \mathrm{IR}, F,[1,2]}$ and $\gamma_{\bar{\psi} \psi}, \mathrm{IR}, F,[0,3]$ are dashed magenta and dotted magenta.

the conformality upper bound yields the new Padé-based estimate of $N_{f, c r}$. For this $\mathrm{SU}(2)$ theory [and for the $\mathrm{SU}(3)$ and SU(4) theories to be discussed below] the larger Padé-based value is $\gamma_{\bar{\psi} \psi, I R, F,[0,3]}$. From Fig. 1, we therefore infer that

$$
\mathrm{SU}(2), R=F: \quad N_{f, c r} \simeq 5-6 .
$$

This result is consistent with a recent lattice study [46] of the $\mathrm{SU}(2)$ theory with $R=F, N_{f}=6$, which finds this theory is IR-conformal. (Earlier lattice studies of this theory include [47].) With this estimate that $N_{f \text {,cr }} \lesssim 6$, it follows that the non-Abelian Coulomb phase occupies the physical interval $6 \leq N_{f} \leq 10$, the same as the interval $I$ with $N_{f} \in \mathbb{N}_{+}$.

As another application, we can calculate Taylor series expansions of these Padé approximants to see what they predict for higher-order coefficients, namely the $\kappa_{j, F}$ with $j \geq 5$. [Recall that the $\kappa_{j, R}$ were given for general $G$ and $R$ in [14-16] and were listed numerically for $G=\mathrm{SU}(2)$, $R=F$ in Eq. (3.6) above.] We find that the Padé approximant $\gamma_{\bar{\psi} \psi, \mathrm{IR}, F,[0,3]}$ that we used to estimate $N_{f, c r}$ yields coefficients $\kappa_{j, F}$ with $j \geq 5$ that are all positive to the highest order to which we have calculated them, namely $j=200$. This is an important result, because it shows that our use of this approximant, $\gamma_{\bar{\psi} \psi, \mathrm{IR}, F,[0,3]}$, to estimate $N_{f, c r}$ is self-consistent. That is, our use assumed that, in addition to the known positive $\kappa_{j, F}$ with $1 \leq j \leq 4$, the higher-order $\kappa_{j}$ with $j \geq 5$ are positive, and our Taylor series expansion of $\gamma_{\bar{\psi} \psi, \mathrm{IR}, F,[0,3]}$ is consistent with this. In Table IV we list the higher-order coefficients $\kappa_{j, F,[0,3]}$ with $5 \leq j \leq 10$ for this theory obtained from the Taylor series expansions of the 
Padé approximant $\gamma_{\bar{\psi} \psi, I R, F,[0,3]}$. Since we did not use the $\gamma_{\bar{\psi} \psi, \mathrm{IR}, F,[2,1]}$ for our estimate of $N_{f, c r}$, it is not of direct relevance what the signs of the $\kappa_{j, F}$ with $j \geq 5$ from the Taylor series expansion of this [2,1] approximant. However, for completeness, we mention that they include both positive and negative ones in an alternating manner. The first few are $\kappa_{5, F,[1,2]}=-\left(0.4344482 \times 10^{-4}\right), \kappa_{6, F,[1,2]}=$ $0.323207 \times 10^{-4}, \kappa_{7, F,[1,2]}=-\left(1.90897 \times 10^{-5}\right)$, etc. This difference in the signs of the $\kappa_{j, F,[0,3]}$ and $\kappa_{j, F,[1,2]}$ for $j \geq 5$ accounts for the fact that $\gamma_{\bar{\psi} \psi, I R, F,[0,3]}>\gamma_{\bar{\psi} \psi, I R, F,[1,2]}$, as observed in Table II and Fig. 1. Similar comments apply for the $\mathrm{SU}(3)$ and $\mathrm{SU}(4)$ theories with $R=F$ to be discussed next.

\section{D. $\mathrm{SU}(3)$}

For $G=\mathrm{SU}(3)$ with $R=F$, the general formulas (2.1) and (2.3) yield the values $N_{u}=16.5, N_{\ell}=8.053$. Thus, for this theory, the interval $I$ for $N_{f} \in \mathbb{R}_{+}$is
$8.053<N_{f}<16.5$ with $\Delta_{f, \max }=8.447$, and the physical interval $I$ with $N_{f} \in \mathbb{N}_{+}$is $9 \leq N_{f} \leq 16$ (see Table I). From our calculation of $\gamma_{\bar{\psi} \psi, \mathrm{IR}, F, \Delta_{f}^{4}}$ in [13], we presented polynomial extrapolations to infinite order to obtain estimates of $\lim _{s \rightarrow \infty} \gamma_{\bar{\psi} \psi} \psi \mathrm{IR}, \Delta_{f}^{s}$. Combining these with the conformality upper bound (2.12) and the assumption that, as in the supersymmetric case, $\gamma_{\bar{\psi} \psi, \mathrm{IR}}$ saturates this upper bound at the lower end of the NACP, we estimated that [13]

$$
\mathrm{SU}(3), \quad R=F: N_{f, c r} \simeq 8-9,
$$

in agreement with several lattice estimates [23,48-54]. As we will discuss, our new calculations presented here are consistent, to within the intrinsic theoretical uncertainties involved, with our estimate of $N_{f, c r}$ given in [13].

For this $\mathrm{SU}(3)$ theory, we calculate the following $[p, q]$ Padé approximants with $q \neq 0$ :

$$
\begin{aligned}
& \gamma_{\bar{\psi} \psi, F,[2,1]}=0.0498442 \Delta_{f}\left[\frac{1-0.0788254 \Delta_{f}-0.00702398 \Delta_{f}^{2}}{1-0.154919 \Delta_{f}}\right], \\
& \gamma_{\bar{\psi} \psi, F,[1,2]}=0.0498442 \Delta_{f}\left[\frac{1+0.442170 \Delta_{f}}{1+0.366077 \Delta_{f}-0.0326204 \Delta_{f}^{2}}\right], \\
& \gamma_{\bar{\psi} \psi, F,[0,3]}=0.0498442 \Delta_{f}\left[\frac{1}{1-0.0760935 \Delta_{f}+\left(1.02588 \times 10^{-3}\right) \Delta_{f}-\left(0.4536614 \times 10^{-3}\right) \Delta_{f}^{3}}\right] .
\end{aligned}
$$

The [2,1] Padé approximant in $\gamma_{\bar{\psi} \psi, F,[2,1]}$ has a pole at $\Delta_{f}=6.455$, i.e., at $N_{f}=10.045$. Hence, this pole lies in the interval $I$ and also in the NACP, so we cannot use this [2,1] Padé approximant for our analysis. The [1,2] Padé approximant in $\gamma_{\bar{\psi} \psi, F,[1,2]}$ has poles at $\Delta_{f}=-2.272$, i.e., $N_{f}=18.772$, and at $\Delta_{f}=13.494$, i.e., $N_{f}=3.006$. The first of these occurs at a value of $N_{f}$ greater than $N_{u}$, while the second occurs at a value of $N_{f}$ well below $N_{\ell}$ and $N_{f, c r}$, so neither is in the interval $I$ or in the NACP. Finally, the $[0,3]$ Padé approximant has a pole at $\Delta_{f}=9.392$, i.e., $N_{f}=7.108$, and a complex-conjugate pair of poles at $\Delta_{f}=-3.565 \pm 14.900 i$. The real pole lies below $N_{\ell}$ and the above-mentioned estimates of the lower end of the NACP at $N_{f}=8-9$, while the complex poles have magnitude 15.321 , which is considerably larger than $\Delta_{f, \max }=8.447$ and $\Delta_{c r} \simeq 8$. Hence, we can make use of both the [1,2] and [0,3] Padé approximants for our analysis.

In Table II we list values of $\gamma_{\bar{\psi} \psi, \mathrm{IR}, F,[1,2]}$ and $\gamma_{\bar{\psi} \psi, \mathrm{IR}, F,[0,3]}$ for this $\mathrm{SU}(3)$ theory as a function of $N_{f}$ and, for comparison, values of $\gamma_{\bar{\psi} \psi, \mathrm{IR}, F, \Delta_{f}^{s}}$ for $1 \leq s \leq 4$ from
[14,16]. In Fig. 2 we plot these two Padé approximants to our $O\left(\Delta_{f}^{4}\right)$ series, $\gamma_{\bar{\psi} \mu \mu, \mathrm{IR}, F,[1,2]}$ and $\gamma_{\bar{\psi} \psi, \mathrm{IR}, F,[0,3]}$, as a function of $N_{f}$ for $N_{f} \in I$. For comparison, we also show our previously calculated $\gamma_{\bar{\psi} \psi, \mathrm{IR}, F, \Delta_{f}^{s}}$ with $1 \leq s \leq 4$.

The general features that we remarked on for the SU(2) theory with $R=F$ are also evident here. For $N_{f}$ in the upper part of the non-Abelian Coulomb phase, the values of $\gamma_{\bar{\psi} \mu, \mathrm{IR}, F,[1,2]}$ and $\gamma_{\bar{\psi} \psi, \mathrm{IR}, F,[0,3]}$ are quite close to $\gamma_{\bar{\psi} \psi, \mathrm{IR}, R, \Delta_{f}^{4}}$. As $N_{f}$ decreases, $\gamma_{\bar{\psi} \psi, \mathrm{IR}, F,[1,2]}$ continues to be close to $\gamma_{\bar{\psi} \mu, \mathrm{IR}, F, \Delta_{f}^{4}}$, while $\gamma_{\bar{\psi} \psi, \mathrm{IR}, R,[0,3]}$ becomes progressively larger than $\gamma_{\bar{\psi} \psi, I R, F, \Delta_{f}^{4}}$ and $\gamma_{\bar{\psi} \psi, I R, F,[1,2]}$. For small $N_{f}$ near to the lower end of the non-Abelian Coulomb phase, $\gamma_{\bar{\psi} \psi, \mathrm{IR}, F,[0,3]}$ rises up and eventually exceeds the conformality upper bound (2.12) for $N_{f}$ between 8 and 9. Using the value of $N_{f}$ where $\gamma_{\bar{\psi} \psi, I R, F,[0,3]}$ exceeds this upper bound as an estimate of $N_{f, c r}$, we derive the result $N_{f, c r} \sim 8-9$, in agreement with Eq. (3.14) from [13] and with most lattice estimates.

Our new results from the Padé approximants extend our previous comparison with lattice measurements in [13-16]. 


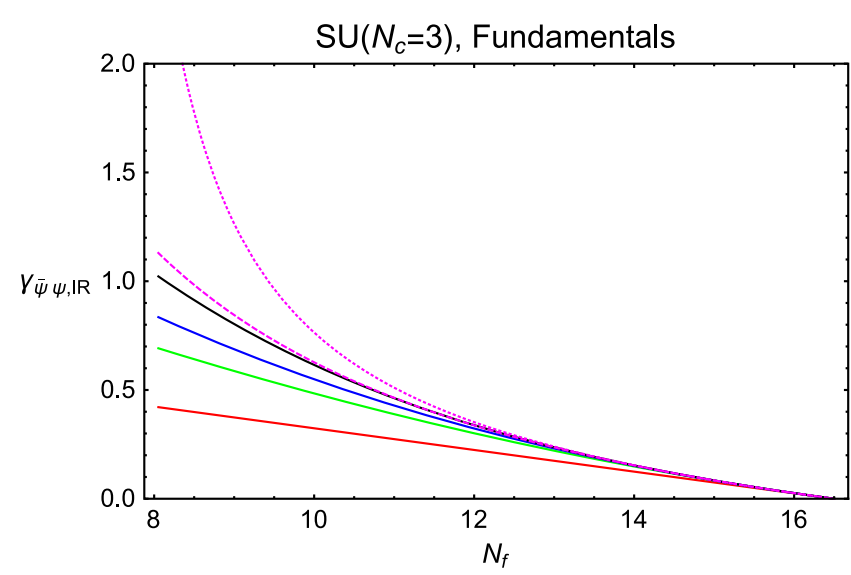

FIG. 2. Plot of $\gamma_{\bar{\psi} \psi, \mathrm{IR}, F,[1,2]}$ and $\gamma_{\bar{\psi} \psi, \mathrm{IR}, F,[0,3]}$ for $N_{c}=3$, i.e., $\mathrm{SU}(3)$ and $R=F$, together with $\gamma_{\bar{\psi} \psi, \mathrm{IR}, F, \Delta_{f}^{s}}$ with $1 \leq s \leq 4$, as a function of $N_{f}$. The vertical axis is labelled generically as $\gamma_{\bar{\psi} \mu, \mathrm{IR}}$. From bottom to top, the curves (with colors online) refer to $\gamma_{\bar{\psi} \psi, \mathrm{IR}, F, \Delta_{f}}$ (red), $\gamma_{\bar{\psi} \psi\left(\mathrm{IR}, F, \Delta_{f}^{2}\right.}$ (green), $\gamma_{\bar{\psi} \psi \psi, \mathrm{IR}, F, \Delta_{f}^{3}}$ (blue), and $\gamma_{\bar{\psi} \psi} \psi, \mathrm{IR}, F, \Delta_{f}^{4}$ (black). The curves for $\gamma_{\bar{\psi} \psi \psi, \mathrm{IR}, F,[1,2]}$ and $\gamma_{\bar{\psi} \psi, \mathrm{IR}, F,[0,3]}$ are dashed magenta and dotted magenta.

There have been a number of studies of the SU(3) theory with $R=F$ and $N_{f}=12$, including [48-55]. Although many lattice groups have concluded that this theory is IR-conformal, there is not yet a consensus on this point (for recent reviews, see [23]). As listed in Table II, rounding off to two significant figures, for this $\mathrm{SU}(3)$ theory with $R=F$ and $N_{f}=12$, our new values from the Padé approximants, namely $\gamma_{\bar{\psi} \psi, \mathrm{IR}, F,[1,2]}=0.34$ and $\gamma_{\bar{\psi} \psi, \mathrm{IR}, F,[0,3]}=0.35$, are in good agreement with our scheme-independent series calculations in [13], namely, $\gamma_{\bar{\psi} \psi, \mathrm{IR}, F, \Delta_{f}^{3}}=0.32$ and $\gamma_{\bar{\psi} \psi, \mathrm{IR}, F, \Delta_{f}^{4}}=0.34$, and are somewhat higher than our conventional $n$-loop calculations, $\quad \gamma_{\bar{\psi} \psi, \mathrm{IR}, F, 3 \ell}=0.31$, $\gamma_{\bar{\psi} \psi, \mathrm{IR}, F, 4 \ell}=0.25$ [2], and $\gamma_{\bar{\psi} \psi, \mathrm{IR}, F, 5 \ell}=0.26$ [13]. The lattice simulations have obtained a range of values for $\gamma_{\bar{\psi} \psi, \mathrm{IR}, F}$, including the following: $\gamma_{\bar{\psi} \psi, \mathrm{IR}, F} \sim 0.414(16)$ [48], $\gamma_{\bar{\psi} \psi, \mathrm{IR}, F} \simeq 0.35$ [49], $\gamma_{\bar{\psi} \psi, \mathrm{IR}, F} \simeq 0.4$ [50], $\gamma_{\bar{\psi} \psi, \mathrm{IR}, F}=0.27(3)$ [51], $\gamma_{\bar{\psi} \psi, \mathrm{IR}, F} \simeq 0.25$ [52] (see also [53]), $\gamma_{\bar{\psi} \psi, \mathrm{IR}, F}=$ $0.235(46)$ [54], and $0.2 \lesssim \gamma_{\bar{\psi} \psi, \mathrm{IR}, F} \lesssim 0.4$ [55]. Here, the quoted uncertainties in the last digits are indicated in parentheses; we refer the reader to these papers for detailed discussions of overall uncertaintites in these measurements. Recent critical discussions of these lattice measurements include $[23,55,56]$.

It is also worthwhile to compare our results with other lattice studies, bearing in mind that (i) our calculations assume an exact IR fixed point, as is true in the nonAbelian Coulomb phase, and (ii) as $N_{f}$ decreases toward the lower end of the NACP and $\Delta_{f}$ increases, one generally needs more terms in a series expansion in powers of $\Delta_{f}$ to achieve a given accuracy. For SU(3),
$R=F$, and $N_{f}=10$ (see Table II), again rounding off to two significant figures, we obtain the Padé approximant values $\gamma_{\bar{\psi} \psi, \mathrm{IR}, F,[1,2]}=0.63$ and $\gamma_{\bar{\psi} \psi, \mathrm{IR}, F,[0,3]}=0.76$. The first of these Padé-based values is close to our highest-order scheme-independent series calculation [13], $\gamma_{\bar{\psi} \psi, I R, F, \Delta_{f}^{4}}=0.62$, while the second is slightly higher. A study of the $\mathrm{SU}(3)$ theory with $R=F$ and $N_{f}=10$ was reported in [57], with the result $\gamma_{\bar{\psi} \psi, \mathrm{IR}, F} \sim O(1)$. To within the estimated uncertainties, our values for this theory from [13], as augmented by our new results from Padé approximants, are in reasonable agreement with this estimate of $\gamma_{\bar{\psi} \psi, \mathrm{IR}, F}$ from [57]. For SU(3) with $R=F$ and $N_{f}=8$, as is evident in Table II, there is a significant difference between the values of our two Padé approximants, indicating that a calculation of the series to higher order in $\Delta_{f}$ than $O\left(\Delta_{f}^{4}\right)$ would be desirable. This theory with $N_{f}=8$ has been the subject of a number of lattice studies, including [58,59] (see also [60,61]), which have observed quasiconformal behavior. This behavior is consistent with the inference that for $\mathrm{SU}(3)$ and $R=F$, the value $N_{f}=8$ is close to, but slightly less than, $N_{f, c r}$, in agreement with our estimate in Eq. (3.14) [25].

The Taylor series expansion of the Padé approximant $\gamma_{\bar{\psi} \psi, \mathrm{IR}, F,[0,3]}$ to calculate higher-order coefficients $\kappa_{j, F}$ with $j \geq 5$ is again of interest for this $\mathrm{SU}(3)$ theory. We list the higher-order coefficients $\kappa_{j, F}$ with $5 \leq j \leq 10$ from the Taylor series expansion of $\gamma_{\bar{\psi} \psi, I R, F,[0,3]}$ in Table IV. As was the case with the SU(2) theory, these coefficients are all positive. We find the same positivity for the highest order, $j=200$, to which we have calculated the series expansion of this Padé approximant in powers of $\Delta_{f}$. As with SU(2), this shows the self-consistency of our use of $\gamma_{\bar{\psi} \psi, \mathrm{IR}, F,[0,3]}$ here to estimate $N_{f, c r}$, which assumed this positivity of higher-order coefficients. Similarly to the SU(2) theory, the higher-order coefficients $\kappa_{j, F}$ from the Taylor series expansion of the other approximant, $\gamma_{\bar{\psi} \psi, \mathrm{IR}, F,[1,2]}$, which we did not use to estimate $N_{f, c r}$ (since it is smaller than use of $\left.\gamma_{\bar{\psi} \psi, I R, F,[0,3]}\right)$ have alternating signs, starting with a negative $\kappa_{5, F}$.

\section{E. SU(4)}

For SU(4) with $R=F$, the general formulas (2.1) and (2.3) yield the values $N_{u}=22, N_{\ell}=10.615$. Thus, for this theory, the interval $I$ with $N_{f} \in \mathbb{R}_{+}$is $10.615<N_{f}<22$, with $\Delta_{f, \max }=11.385$, and the physical interval $I$ with $N_{f} \in \mathbb{N}_{+}$is $11 \leq N_{f} \leq 21$.

For this SU(4) theory, we calculate the following $[p, q]$ Padé approximants with $q \neq 0$ : 


$$
\begin{aligned}
& \gamma_{\bar{\psi} \psi, F,[2,1]}=0.0385604 \Delta_{f}\left[\frac{1-0.0547830 \Delta_{f}-\left(0.360664 \times 10^{-2}\right) \Delta_{f}^{2}}{1-0.112651 \Delta_{f}}\right], \\
& \gamma_{\bar{\psi} \psi, F,[1,2]}=0.0385604 \Delta_{f}\left[\frac{1+0.423414 \Delta_{f}}{1+0.365546 \Delta_{f}-0.0240657 \Delta_{f}^{2}}\right], \\
& \gamma_{\bar{\psi} \psi, F,[0,3]}=0.0385604 \Delta_{f}\left[\frac{1}{1-0.05786815 \Delta_{f}+\left(0.436451 \times 10^{-3}\right) \Delta_{f}-\left(1.847995 \times 10^{-4}\right) \Delta_{f}^{3}}\right] .
\end{aligned}
$$

The [2,1] Padé approximant in $\gamma_{\bar{\psi} \psi, F,[2,1]}$ has a pole at $\Delta_{f}=8.877$, i.e., at $N_{f}=13.123$. Since this pole lies in the interval $I$, we do not use the $[2,1]$ Padé approximant. The [1,2] Padé approximant in $\gamma_{\bar{\psi} \psi, F,[1,2]}$ has poles at $\Delta_{f}=-2.367$, i.e., $N_{f}=24.367$, and at $\Delta_{f}=17.556$, i.e., $N_{f}=4.444$. The first of these occurs at a value of $N_{f}$ greater than $N_{u}$, while the second occurs at a value of $N_{f}$ well below $N_{\ell}$. Finally, the [0,3] Padé has a pole at $\Delta_{f}=12.379$, i.e., $N_{f}=9.621$, which is below $N_{\ell}$. This [0,3] approximant also has complex poles at $\Delta_{f}=$ $-5.0085 \pm 20.299 i$, of magnitude 20.908 , which is considerably greater than $\Delta_{f, \max }=11.385$ and $\Delta_{f, c r} \simeq 11$. We can thus use both the [1,2] and [0,3] Padé approximants for our analysis. This is the same set that we could use in the case of the $\mathrm{SU}(2)$ and $\mathrm{SU}(3)$ gauge theories with $R=F$.

In Table II we list the values of the Padé approximants $\gamma_{\bar{\psi} \psi, \mathrm{IR}, F,[1,2]}$ and $\gamma_{\bar{\psi} \psi, \mathrm{IR}, F,[0,3]}$ for this $\mathrm{SU}(4)$ theory, as a function of $N_{f}$. For comparison, we also include the values of $\gamma_{\bar{\psi} \psi, \mathrm{IR}, F, \Delta_{f}^{s}}$ with $1 \leq s \leq 4$ from [14,16]. In Fig. 3 we plot all of these values. The general features of these results for SU(4) are similar to the features that we have already discussed for $\mathrm{SU}(2)$ and $\mathrm{SU}(3)$. Again using the larger of the two Padé-based values, $\gamma_{\bar{\psi} \psi, \mathrm{IR}, F,[0,3]}$, and inferring $N_{f, c r}$ as the value of $N_{f}$ where this exceeds the conformality upper bound, we derive the estimate

$$
\mathrm{SU}(4): \quad N_{f, c r} \sim 11 .
$$

The self-consistency of our procedure is again shown by the fact that the higher-order coefficients $\kappa_{j, F}$ with $j \geq 5$ from the Taylor series expansion of $\gamma_{\bar{\psi} \psi, \mathrm{IR}, F,[0,3]}$ are positive. We list these $\kappa_{j, F}$ in Table IV.

\section{F. LNN limit}

Here, in the LNN limit, we calculate Padé approximants for our series $\gamma_{\bar{\psi} \psi, \mathrm{IR}, \Delta_{r}^{4}}$ from $s=4$ in $[14,16]$. The values of $\hat{\kappa}_{j, F}$ were given (analytically) in [14] for $1 \leq j \leq 3$ and in [16] for $j=4$, and are

$$
\begin{aligned}
\hat{\kappa}_{1, F} & =\frac{2^{2}}{5^{2}}=0.1600, \\
\hat{\kappa}_{2, F} & =\frac{588}{5^{6}}=0.037632, \\
\hat{\kappa}_{3, F} & =\frac{2193944}{3^{3} \cdot 5^{10}}=0.83207 \times 10^{-2}, \\
\hat{\kappa}_{4, F} & =\frac{210676352}{3^{4} \cdot 5^{13}}+\frac{90112}{3^{3} \cdot 5^{10}} \zeta_{3}+\frac{11264}{3^{3} \cdot 5^{8}} \zeta_{5} \\
& =0.36489 \times 10^{-2} \cdot
\end{aligned}
$$

The resultant $\gamma_{\bar{\psi} \psi, \mathrm{IR}, F, L N N, \Delta_{r}^{4}}$ is

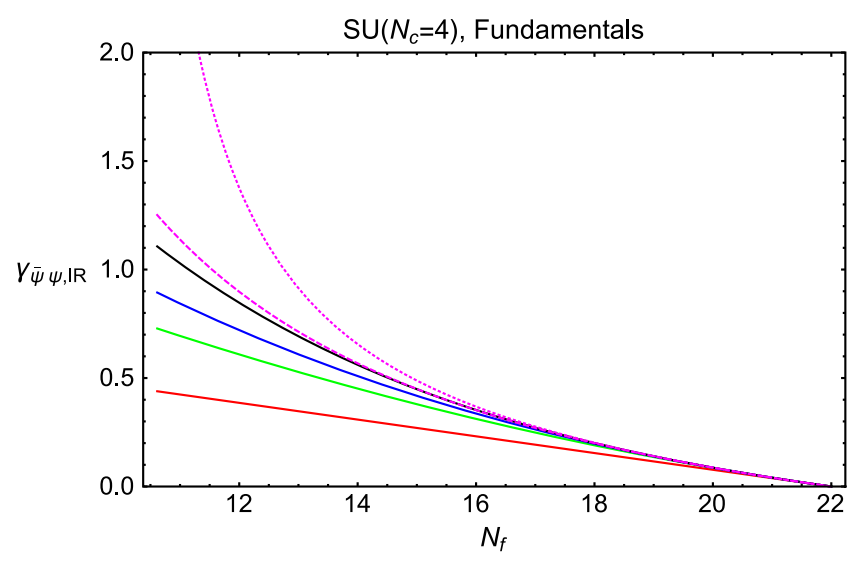

FIG. 3. Plot of $\gamma_{\bar{\psi} \psi, \mathbb{R}, F,[1,2]}$ and $\gamma_{\bar{\psi} \psi,[R, F,[0,3]}$ for $N_{c}=4$, i.e., $\mathrm{SU}(4)$ and $R=F$, together with $\gamma_{\bar{\psi} \psi, \mathrm{IR}, F, \Delta_{f}^{s}}$ with $1 \leq s \leq 4$, as a function of $N_{f}$. The vertical axis is labelled generically as $\gamma_{\bar{\psi} \psi, \mathrm{IR}}$. From bottom to top, the curves (with colors online) refer to $\gamma_{\bar{\psi} \psi, I R, F, \Delta_{f}}$ (red), $\gamma_{\bar{\psi} \psi, I R, F, \Delta_{f}^{2}}$ (green), $\gamma_{\bar{\psi} \psi, I R, F, \Delta_{f}^{3}} \quad$ (blue), and $\gamma_{\bar{\psi} \psi, I \mathbb{R}, F, \Delta_{f}^{4}}$ (black). The curves for $\gamma_{\bar{\psi} \psi, \mathbb{R}, F,[1,2]}$ and $\gamma_{\bar{\psi} \psi, \mathrm{IR}, F,[0,3]}$ are dashed magenta and dotted magenta. 


$$
\begin{aligned}
& \text { LNN }, \quad R=F \text { : } \\
& \gamma_{\bar{\psi} \psi, \mathrm{IR}, F, L N N, \Delta_{r}^{4}}=0.160000 \Delta_{f}+0.0376320 \Delta_{r}^{2} \\
& +\left(0.832074 \times 10^{-2}\right) \Delta_{r}^{3} \\
& +\left(0.364894 \times 10^{-2}\right) \Delta_{r}^{4} \text {. }
\end{aligned}
$$

In Eq. (3.26) we have listed $\gamma_{\bar{\psi} \psi, \mathrm{IR}, F, L N N, \Delta_{r}^{4}}$ in numerical form, to the indicated floating-point accuracy, but in our actual computer calculations, the coefficients are used to considerably higher accuracy.

Analogously to Eq. (3.1), we write

$$
\begin{aligned}
\gamma_{\bar{\psi} \mu, \mathrm{IR}, F, L N N, \Delta_{r}^{s}} & =\sum_{j=1}^{s} \hat{\kappa}_{j, F} \Delta_{r}^{j} \\
& =\hat{\kappa}_{1, F} \Delta_{r}\left[1+\frac{1}{\hat{\kappa}_{1, F}} \sum_{j=2}^{s} \hat{\kappa}_{j, F} \Delta_{r}^{j-1}\right]
\end{aligned}
$$

and calculate the $[p, q]$ Padé approximant to the expression in square brackets, with $p+q=s-1$. We have calculated analytic results for Padé approximants to $\gamma_{\bar{\psi} \mu, \mathrm{IR}, F, \Delta_{r}^{4}}$. It is again simplest to present these in numerical form.

We find

$$
\begin{aligned}
& \gamma_{\bar{\psi} \psi, \mathrm{IR}, F, L N N,[2,1]}=\frac{4}{25} \Delta_{r}\left[\frac{1-0.203335 \Delta_{r}-0.0511389 \Delta_{r}^{2}}{1-0.438535 \Delta_{r}}\right], \\
& \gamma_{\bar{\psi} \psi, \mathrm{IR}, F, L N N,[1,2]}=\frac{4}{25} \Delta_{r}\left[\frac{1+3.425595 \Delta_{r}}{1+3.190395 \Delta_{r}-0.80238556 \Delta_{r}^{2}}\right], \\
& \gamma_{\bar{\psi} \psi, \mathrm{IR}, F, L N N,[0,3]}=\frac{4}{25} \Delta_{r}\left[\frac{1}{1-0.235200 \Delta_{r}+0.00331444 \Delta_{r}^{2}-0.0113539 \Delta_{r}^{3}}\right] .
\end{aligned}
$$

The [2,1] Padé approximant has a pole at $\Delta_{r}=2.28032$, or equivalently, $r=3.21968$, which lies in the interval $I_{r}$ and also in the inferred NACP [see Eq. (2.21) below], and hence we cannot use this approximant for our analysis. The [1,2] Padé approximant has poles at $\Delta_{r}=-0.291997$ and $\Delta_{r}=4.26813$, i.e., at $r=5.79200$ and $r=1.23187$. The first pole lies above $r_{u}$, where the theory is not asymptotically free, and the second pole lies below $r_{\ell}$. Considering $\gamma_{\bar{\psi} \psi, I R, F, L N N,[1,2]}$ as an analytic function, the pole at $\Delta_{r}=-0.291997$ lies much closer to the origin $\Delta_{r}=0$ than the radii of both of the disks $\left|\Delta_{r}\right|<\Delta_{r, \text { max }}=$ 2.8846 and $\left|\Delta_{r}\right|<\Delta_{r, c r}=2.6$ [where $\Delta_{r, c r}$ is given below in Eq. (2.24)]. Consequently, we cannot use this [1,2] Padé fully reliably for our analysis. However, it turns out that because the pole lies on the opposite side of the origin in the $\Delta_{r}$ plane, at negative $\Delta_{f}$, relative to the interval $I: 0<\Delta_{r}<2.8846$ and the NACP, where $0<\Delta_{r} \lesssim 2.9$, the approximant $\gamma_{\bar{\psi} \psi, I R, F, L N N,[1,2]}$ is actually rather close to $\gamma_{\bar{\psi} \psi, \mathrm{IR}, F, \Delta_{r}^{4}}$. The $[0,3]$ Padé approximant has a pole at $\Delta_{r}=3.03365$, which is larger than $\Delta_{r, \max }$ and $\Delta_{r, c r}$. In terms of $r$, this pole is at $r=2.46635$, which lies below $r_{\ell}$. This [0,3] approximant also has complex poles at $\Delta_{r}=$ $-1.370865 \pm 5.210899 i$ with magnitude $\left|\Delta_{r}\right|=5.38820$, which is larger than the values $\Delta_{r, \max }=2.8846$ and $\Delta_{r, c r}=2.6$. Therefore, we can use the [0,3] Padé approximant reliably.

In Table III we list values of $\gamma_{\bar{\psi} \psi, \mathrm{IR}, F,[1,2]}$ and $\gamma_{\bar{\psi} \psi}, \mathrm{IR}, F,[0,3]$ for this LNN limit and, for comparison, values of $\gamma_{\bar{\psi} \psi, \mathrm{IR}, F, \Delta_{r}^{s}}$ for $1 \leq s \leq 4$ from $[14,16]$. We have remarked above that although one of the poles in $\gamma_{\bar{\psi} \psi, \mathrm{IR}, F,[1,2]}$ lies within the disk $\left|\Delta_{r}\right|<\Delta_{r, \max }$ in the complex $\Delta_{r}$ plane, this pole does not occur in the region of positive $\Delta_{r}$ of interest here and hence

TABLE III. Values of $\gamma_{\bar{\psi} \psi, I R, F,[p, q]}$ and, for comparison, values of $\gamma_{\bar{\psi} \psi} \psi, \mathrm{IR}, F, \Delta_{r}^{s}$ with $1 \leq s \leq 4$, in the LNN limit (2.15). Values of anomalous dimensions that exceed the conformality upper bound $\gamma_{\bar{\psi} \psi \text {,IR }} \leq 2$ are marked with brackets. To save space, we omit the $\bar{\psi} \psi$ from the subscripts in the table, writing $\gamma_{\bar{\psi} \bar{\psi} \psi \mathrm{IR}, F, \Delta_{r}^{s}} \equiv \gamma_{\mathrm{IR}, F, \Delta_{r}^{s}}$, etc.

\begin{tabular}{llllllc}
\hline \hline$r$ & $\gamma_{\mathrm{IR}, F, \Delta_{r}}$ & $\gamma_{\mathrm{IR}, F, \Delta_{r}^{2}}$ & $\gamma_{\mathrm{IR}, F, \Delta_{r}^{3}}$ & $\gamma_{\mathrm{IR}, F, \Delta_{r}^{4}}$ & $\gamma_{\mathrm{IR}, F,[1,2]}$ & $\gamma_{\mathrm{IR}, F,[0,3]}$ \\
\hline 2.8 & 0.432 & 0.706 & 0.870 & 1.064 & 1.176 & {$[2.608]$} \\
2.9 & 0.416 & 0.670 & 0.817 & 0.983 & 1.065 & 1.968 \\
3.0 & 0.400 & 0.635 & 0.765 & 0.908 & 0.966 & 1.567 \\
3.2 & 0.368 & 0.567 & 0.668 & 0.770 & 0.798 & 1.087 \\
3.4 & 0.336 & 0.502 & 0.579 & 0.650 & 0.662 & 0.809 \\
3.6 & 0.304 & 0.440 & 0.497 & 0.5445 & 0.548 & 0.624 \\
3.8 & 0.272 & 0.381 & 0.422 & 0.452 & 0.452 & 0.491 \\
4.0 & 0.240 & 0.325 & 0.353 & 0.371 & 0.370 & 0.389 \\
4.2 & 0.208 & 0.272 & 0.290 & 0.300 & 0.299 & 0.308 \\
4.4 & 0.176 & 0.2215 & 0.233 & 0.238 & 0.237 & 0.241 \\
4.6 & 0.144 & 0.1745 & 0.1805 & 0.183 & 0.1825 & 0.184 \\
4.8 & 0.112 & 0.130 & 0.133 & 0.134 & 0.134 & 0.134 \\
5.0 & 0.0800 & 0.0894 & 0.09045 & 0.0907 & 0.0906 & 0.0907 \\
5.2 & 0.0480 & 0.0514 & 0.0516 & 0.0516 & 0.0516 & 0.0516 \\
5.4 & 0.0160 & 0.0164 & 0.0164 & 0.0164 & 0.0164 & 0.0164 \\
\hline \hline
\end{tabular}


TABLE IV. Values of higher-order $\kappa_{j, F,[0,3]}$ with $5 \leq j \leq 10$ obtained by Taylor series expansions of $\gamma_{\bar{\psi} \psi}, F,[0,3]$ for $\mathrm{SU}\left(N_{c}\right)$ theories with $N_{c}=2,3,4$. The notation $a$ e-n means $a \times 10^{-n}$.

\begin{tabular}{llll}
\hline \hline$j$ & $\kappa_{j, F,[0,3], \mathrm{SU}(2)}$ & $\kappa_{j, F,[0,3], \mathrm{SU}(3)}$ & $\kappa_{j, F,[0,3], \mathrm{SU}(4)}$ \\
\hline 5 & $2.876447 \mathrm{e}-5$ & $0.427629 \mathrm{e}-5$ & $1.095416 \mathrm{e}-6$ \\
6 & $3.721786 \mathrm{e}-6$ & $0.395378 \mathrm{e}-6$ & $0.7862105 \mathrm{e}-7$ \\
7 & $0.606848 \mathrm{e}-6$ & $0.423869 \mathrm{e}-7$ & $0.640937 \mathrm{e}-8$ \\
8 & $1.056182 \mathrm{e}-7$ & $0.475954 \mathrm{e}-8$ & $0.539017 \mathrm{e}-9$ \\
9 & $1.625598 \mathrm{e}-8$ & $0.498035 \mathrm{e}-9$ & $0.429237 \mathrm{e}-10$ \\
10 & $2.5266295 \mathrm{e}-9$ & $0.522418 \mathrm{e}-10$ & $0.343311 \mathrm{e}-11$ \\
\hline \hline
\end{tabular}

does not strongly affect the values of $\gamma_{\bar{\psi} \psi, \mathrm{IR}, F,[1,2]}$ in this region. As was the case with the specific $\mathrm{SU}\left(N_{c}\right)$ theories with $R=F$ discussed above, for each of the given $r$ values in Table III, $\gamma_{\bar{\psi} \psi \mu, \mathrm{IR}, F,[0,3]}$ is larger than $\gamma_{\bar{\psi} \psi}, \mathrm{IR}, F,[1,2]$. At the upper end of the NACP, these are very close to each other, as they are to $\gamma_{\bar{\psi} \psi}, F, \Delta_{r}^{4}$. As $r$ decreases sufficiently, $\gamma_{\bar{\psi} \psi, \mathrm{IR}, F,[0,3]}$ first exceeds the conformality upper bound at $r \simeq 2.9$. Thus, if we use this value as the estimate of the lower end of the NACP, we infer that

$$
L N N: \quad r_{c r} \simeq 2.9
$$

and hence

$$
L N N: \quad \Delta_{r, c r} \simeq 2.6 .
$$

Our inferred value of $r_{c r}$ defining the lower end of the NACP is slightly larger than the value of $r$ defining the lower end of the interval $I$, namely, $r_{\ell}=2.615$, and correspondingly, the maximal value of $\Delta_{r}$ in the NACP, $\Delta_{r, c r} \simeq 2.6$, is slightly smaller than the maximal value of $\Delta_{r}$ in the interval $I$, namely $\Delta_{r, \max }=2.8846$, as given in (2.23).

It is of interest to investigate how close the $N_{f, c r}$ values inferred for $\mathrm{SU}\left(N_{c}\right)$ theories with $R=F$ and finite $N_{c}$ and $N_{f}$ are to our result (3.31) in the LNN limit. To make this comparison, we compute LNN-based reference ( $L N N r$ ) values, defined as

$$
N_{f, c r, L N N r} \equiv r_{c r} N_{c} .
$$

One does not, a priori, expect very precise agreement between the $N_{f, c r}$ values for finite (and rather small) $N_{c}$ with $N_{f, c r, L N N r}$, since these $N_{c}$ are far from the LNN limit $N_{c} \rightarrow \infty$. Taking the central value in Eq. (3.31), we have

$$
\begin{array}{cl}
\mathrm{SU}(2): & N_{f, c r, L N N r}=5.8, \\
\mathrm{SU}(3): & N_{f, c r, L N N r}=8.7, \\
\mathrm{SU}(4): & N_{f, c r, L N N r}=11.6 .
\end{array}
$$

These are all in approximate agreement with the estimates of $N_{f, c r}$ in Eqs. (3.13), (3.14), and (3.21) given above. This
TABLE V. Values of higher-order $\hat{\kappa}_{j, F, L N N,[0,3]}$ obtained by Taylor series expansions of $\gamma_{\bar{\psi} \psi, F, L N N,[0,3]}$ in the LNN limit. The notation $a$ e-n means $a \times 10^{-n}$.

\begin{tabular}{cc}
\hline \hline$j$ & $\hat{\kappa}_{j, F, L N N,[0,3]}$ \\
\hline 5 & $1.257923 \mathrm{e}-3$ \\
6 & $0.378242 \mathrm{e}-3$ \\
7 & $1.262231 \mathrm{e}-4$ \\
8 & $0.427164 \mathrm{e}-4$ \\
9 & $1.392391 \mathrm{e}-5$ \\
10 & $0.456625 \mathrm{e}-5$ \\
\hline \hline
\end{tabular}

shows that the approach to the LNN limit appears to be rather rapid, even for relatively small values of $N_{c}$.

In Table $\mathrm{V}$ we list the higher-order $\kappa_{j, F, L N N,[0,3]}$ with $5 \leq j \leq 10$ calculated via a Taylor series expansion of $\gamma_{\bar{\psi} \psi, F, L N N,[0,3]}$. We focus on this approximant, since it is the only one free from poles in the disks $\left|\Delta_{r}\right|<\Delta_{r \text {,max }}$ and $\left|\Delta_{r}\right|<\Delta_{r, c r}$. As was the case with the specific $\mathrm{SU}\left(N_{c}\right)$ theories with $R=F$ that we have studied above, all of these higher-order coefficients are positive. We have further verified this positivity up to a much higher order, $j=200$.

\section{G. Adjoint representation}

For $\mathrm{SU}\left(N_{c}\right)$ and $R=A$, the adjoint representation, the values of $N_{\ell}, N_{u}$, and $\Delta_{f, \max }$ were given above in Eq. (2.8). As indicated in Table I, there is only a single integral value of $N_{f}$ in the interval $I$ for these theories, namely $N_{f}=2$. The SU(2) theory with $R=A$ and $N_{f}=2$ has been of some previous theoretical interest $[62,63]$. As we have discussed before [2], since this is a real representation, one could also take $N_{f}$ to be half-integral, corresponding to Majorana fermions, but the $N_{f}=2$ value will be sufficient for our study here. In [2] we carried out $n$-loop calculations of $\gamma_{\bar{\psi} \psi, \mathrm{IR}, n \ell}$ for $2 \leq n \leq 4$. Our two highest-order values for $\mathrm{SU}(2)$ and $\mathrm{SU}(3)$ were

$$
\begin{aligned}
\mathrm{SU}(2), N_{f}=2: & \gamma_{\mathrm{IR}, A, 3 \ell}=0.543, \\
& \gamma_{\mathrm{IR}, A, 4 \ell}=0.500,
\end{aligned}
$$

and

$$
\begin{aligned}
\mathrm{SU}(3), N_{f}=2: & \gamma_{\mathrm{IR}, A, 3 \ell}=0.543, \\
& \gamma_{\mathrm{IR}, A, 4 \ell}=0.523 .
\end{aligned}
$$

We gave explicit analytic results for the scheme-independent expansion coefficients $\kappa_{j, A}$ with $1 \leq j \leq 3$ in [14] and for $j=4$ in [15]. Both $\kappa_{1, A}=4 / 9$ and $\kappa_{2, A}=341 / 1458$ are independent of $N_{c}$, while the $k_{j, A}$ for $j \geq 3$ depend on $N_{c}$. With the prefactor $\kappa_{1, A} \Delta_{f}$ extracted and the Padé approximants normalized to unity at $\Delta_{f}=0$ as in Eq. (3.2), we calculate the following approximants to $\gamma_{\bar{\psi} \psi, I R, A, \Delta_{f}^{4}}$ (in addition to $\left.\gamma_{\bar{\psi} \mu, \mathrm{IR}, A,[3,0]}=\gamma_{\bar{\psi} \mu, \mathrm{IR}, A, \Delta_{f}^{4}}\right)$. 
For $\mathrm{SU}(2)$ we find

$$
\begin{aligned}
& \gamma_{\bar{\psi} \psi, \mathrm{IR}, A,[2,1]}=\frac{4}{9} \Delta_{f}\left[\frac{1-0.793689 \Delta_{f}-0.450643 \Delta_{f}^{2}}{1-1.319924 \Delta_{f}}\right] \\
& \gamma_{\bar{\psi} \psi, \mathrm{IR}, A,[1,2]}=\frac{4}{9} \Delta_{f}\left[\frac{1+6.397597 \Delta_{f}}{1+5.871362 \Delta_{f}-3.333660 \Delta_{f}^{2}}\right] \\
& \gamma_{\bar{\psi} \psi, \mathrm{IR}, A,[0,3]}=\frac{4}{9} \Delta_{f}\left[\frac{1}{1-0.526235 \Delta_{f}+0.0329766 \Delta_{f}^{2}-0.210971 \Delta_{f}^{3}}\right] .
\end{aligned}
$$

Both the $[2,1]$ and $[0,3]$ Padé approximants have poles in the interval $I$, so we do not use them. The [1,2] Padé approximant has poles at $\Delta_{f}=-0.1564$ and $\Delta_{f}=1.918$, i.e., at $N_{f}=2.906$ and $N_{f}=0.8323$, respectively. The first of these poles lies above $N_{u}$, and the second lies below $N_{\ell}$, so we can use this approximant. We list the resultant value, $\gamma_{\bar{\psi} \psi, \mathrm{IR}, A,[1,2]}=0.548$ for $N_{f}=2$ in Table VI. Both this value from the [1,2] Padé approximant and the values $\gamma_{\bar{\psi} \psi, \mathrm{IR}, A, \Delta_{f}^{s}}$ with $s=3$ and $s=4$ are close to our previous higher-order $n$-loop calculations presented in [2], given above in Eq. (3.37). All of these values, which are in accord with each other, are well below the conformality upper limit $\gamma_{\bar{\psi} \psi, \text { IR }} \leq 2$.

For $\mathrm{SU}(3)$ we calculate

$$
\begin{aligned}
& \gamma_{\bar{\psi} \psi, \mathrm{IR}, A,[2,1]}=\frac{4}{9} \Delta_{f}\left[\frac{1-0.434579 \Delta_{f}-0.233470 \Delta_{f}^{2}}{1-0.960813 \Delta_{f}}\right], \\
& \gamma_{\bar{\psi} \psi, \mathrm{IR}, A,[1,2]}=\frac{4}{9} \Delta_{f}\left[\frac{1+25.270167 \Delta_{f}}{1+24.743932 \Delta_{f}-13.293256 \Delta_{f}^{2}}\right], \\
& \gamma_{\bar{\psi} \psi, \mathrm{IR}, A,[0,3]}=\frac{4}{9} \Delta_{f}\left[\frac{1}{1-0.526235 \Delta_{f}+0.00477966 \Delta_{f}^{2}-0.120783 \Delta_{f}^{3}}\right] .
\end{aligned}
$$

Again, both the [2,1] and [0,3] Padé approximants have poles in the interval $I$. The $[1,2]$ Padé approximant has poles at $\Delta_{f}=-0.3957$ and $\Delta_{f}=1.901$, i.e., at $N_{f}=$ 2.790 and $N_{f}=0.8490$. As before, the first of these lies above $N_{u}$ and the second lies below $N_{\ell}$, so we use this approximant. In Table VI we list the value of $\gamma_{\bar{\psi} \mu, \mathrm{IR}, A,[1,2]}=$ 0.551 for this $\mathrm{SU}(3)$ theory with $R=A, N_{f}=2$. As was the case with $\mathrm{SU}(2)$, both this value from the [1,2] Padé approximant and the values $\gamma_{\bar{\psi} \psi, \operatorname{IR}, A, \Delta_{f}^{s}}$ with $s=3$ and $s=4$

TABLE VI. Values of $\gamma_{\bar{\psi} \mu, A, \mathrm{IR},[1,2]}$ and, for comparison, $\gamma_{\bar{\psi} \psi} \psi, A, \mathrm{IR}, \Delta_{f}^{s}$ with $1 \leq s \leq 4$, for $N_{c}=2,3, R=A$ (adjoint), and $N_{f}=2$. To save space, we omit the $\bar{\psi} \psi$ from the subscripts in the table, writing $\gamma_{\bar{\psi} \psi, \mathrm{IR}, A, \Delta_{f}^{s}} \equiv \gamma_{\mathrm{IR}, A, \Delta_{f}^{s}}$, etc.

\begin{tabular}{lcccccc}
\hline \hline$N_{c}$ & $N_{f}$ & $\gamma_{\mathrm{IR}, A, \Delta_{f}}$ & $\gamma_{\mathrm{IR}, A, \Delta_{f}^{2}}$ & $\gamma_{\mathrm{IR}, A, \Delta_{f}^{3}}$ & $\gamma_{\mathrm{IR}, A, \Delta_{f}^{4}}$ & $\gamma_{\mathrm{IR},[1,2]}$ \\
\hline 2 & 2 & 0.333 & 0.465 & 0.511 & 0.556 & 0.548 \\
3 & 2 & 0.333 & 0.465 & 0.516 & 0.553 & 0.551 \\
\hline \hline
\end{tabular}

are close to our previous higher-order $n$-loop calculations presented in [2] and listed above in Eq. (3.38). As was true in the $\mathrm{SU}(2)$ case, all of these values are well below the conformality upper limit $\gamma_{\bar{\psi} \psi, \text { IR }} \leq 2$.

From these results, we infer that the $\mathrm{SU}(2)$ and $\mathrm{SU}(3)$ theories with $R=A$ and $N_{f}=2$ are in the non-Abelian Coulomb phase, and that $N_{f, c r}$ lies below $N_{f}=2$ for these theories. There have been several lattice studies of the $\mathrm{SU}(2)$ theory with $R=A$ and $N_{f}=2$, and these have also concluded that this theory is IR-conformal [23]. A comparison of the values of $\gamma_{\bar{\psi} \psi, I R, A}$ from these lattice measurements with our values from our $\gamma_{\bar{\psi} \psi, I R, A, \Delta_{f}^{4}}$ calculation was made in $[15,16]$. We found that our $\gamma_{\bar{\psi} \psi, \mathrm{IR}, \Delta_{f}^{4}}$ value was in agreement with several of the lattice measurements, but noted that the lattice measurements span a large range. These lattice results for $\gamma_{\bar{\psi} \psi, I R, A}$ include the values $0.49(13)$ [64], 0.22(6) [65], 0.31(6) [66], 0.17(5) [67], 0.50(26) [68], and 0.20(3) [69]. (See these references for details of the uncertainty estimates.) A recent work [70] reported that the 
value of $\gamma_{\bar{\psi} \psi, \text { IR }}$ that was obtained depended on a lattice parameter (the coefficient $\beta$ of the plaquette term in the lattice action) and hence more work was needed to determine the actual $\gamma_{\bar{\psi} \psi, \text { IR }}$.

\section{H. Symmetric rank-2 tensor representation}

For our $\mathrm{SU}\left(N_{c}\right)$ theories with $R=S_{2}$, the symmetric, rank-2 tensor representation, the values of $N_{\ell}, N_{u}$, and $\Delta_{f, \max }$ were given above in Eq. (2.9) and are listed numerically in Table I. In the case of $\mathrm{SU}(2)$, the $S_{2}$ representation is the same as the adjoint representation, which we have already analyzed above. Thus, as in our earlier work, we focus on the two illustrative theories, SU(3) and SU(4). With both of these theories, the interval $I$ contains two integral values of $N_{f}$, namely $N_{f}=2$ and $N_{f}=3$. Explicit analytic results for $\kappa_{j, S_{2}}$ in $\mathrm{SU}\left(N_{c}\right)$ theories with $R=S_{2}$ were given for $1 \leq j \leq 3$ in [14] and for $j=4$ in [16]. The lowest-order coefficient is

$$
\kappa_{1, S_{2}}=\frac{4\left(N_{c}-1\right)\left(N_{c}+2\right)^{2}}{N_{c}\left(18 N_{c}^{2}+11 N_{c}-22\right)} .
$$

This has the values $\kappa_{1, S_{2}}=200 / 519=0.385356$ for $\mathrm{SU}(3)$ and $\kappa_{1, S_{2}}=54 / 155=0.348387$ for SU(4).

With the prefactor $\kappa_{1, S_{2}} \Delta_{f}$ extracted and the Padé approximant normalized as in Eq. (3.2), we calculate the following Padé approximants, in addition to $\gamma_{\bar{\psi} \psi, \mathrm{IR}, S_{2},[3,0]}=$ $\gamma_{\bar{\psi} \mu \psi, I R, S_{2}, \Delta_{f}^{4}}$. For SU(3) we obtain

$$
\begin{aligned}
& \gamma_{\bar{\psi} \psi, \mathrm{IR}, S_{2},[2,1]}=\frac{200}{519} \Delta_{f}\left[\frac{1-0.327520 \Delta_{f}-0.137718 \Delta_{f}^{2}}{1-0.769654 \Delta_{f}}\right] \\
& \gamma_{\bar{\psi} \psi, \mathrm{IR}, S_{2},[1,2]}=\frac{200}{519} \Delta_{f}\left[\frac{1-8.916418 \Delta_{f}}{1-9.358552 \Delta_{f}+3.935165 \Delta_{f}^{2}}\right], \\
& \gamma_{\bar{\psi} \psi, \mathrm{IR}, S_{2},[0.3]}=\frac{200}{519} \Delta_{f}\left[\frac{1}{1-0.442134 \Delta_{f}-0.00708939 \Delta_{f}^{2}-0.0632120 \Delta_{f}^{2}}\right] .
\end{aligned}
$$

All of these three Padé approximants with $q \neq 0$ for the $\mathrm{SU}(3)$ theory with $R=S_{2}$ have poles in the interval $I$. For the corresponding SU(4) theory, we find that the Padé approximants share the property with our SU(3) results of all having poles in the interval $I$. Consequently, for these theories, our Padé analysis does not add to our previous study of the $\gamma_{\bar{\psi} \psi, I R, S_{2}}$ to $O\left(\Delta_{f}^{3}\right)$ in [14] and to $O\left(\Delta_{f}^{4}\right)$ in $[15,16]$. There have been several lattice studies of the SU(3) theory with $N_{f}=2$ fermions in the symmetric rank-2 tensor (sextet) representation. These include Ref. [71], which concluded that it is IR-conformal and obtained $\gamma_{\mathrm{IR}}<0.45$ and Ref. [72], which concluded that it is not IR-conformal, but instead exhibits spontaneous chiral symmetry breaking and obtained an effective $\gamma_{\mathrm{IR}} \simeq 1$.

\section{PADÉ APPROXIMANTS FOR $\beta_{\text {IR }}^{\prime}$ USING THE $O\left(\Delta_{f}^{\mathbf{5}}\right)$ SERIES}

\section{A. General}

In this section we report our computation and analysis of Padé approximants for $\beta_{\mathrm{IR}}^{\prime}$, using our calculation of $\beta_{\mathrm{IR}}^{\prime}$ to $O\left(\Delta_{f}^{4}\right)$ in [14] and to $O\left(\Delta_{f}^{5}\right)$ in $[15,16]$. As noted, $\beta_{\mathrm{IR}}^{\prime}$ is equivalent to the anomalous dimension of $\operatorname{Tr}\left(F_{\mu \nu} F^{\mu \nu}\right)$ $[10,11]$. We also discuss the behavior of $\beta_{\mathrm{IR}}^{\prime}$ toward the lower end of the non-Abelian Coulomb phase at $N_{f}=N_{f, c r}$. This behavior is relevant to the change in the properties of the theory as $N_{f}$ increases through $N_{f, c r}[73]$.

For a given truncation of the series (2.14) to maximal order $s$ we write $\beta_{\mathrm{IR}}^{\prime}$ as

$$
\beta_{\mathrm{IR}, \Delta_{f}^{s}}^{\prime}=\sum_{j=2}^{s} d_{j} \Delta_{f}^{j}=d_{2} \Delta_{f}^{2}\left[1+\frac{1}{d_{2}} \sum_{j=3}^{s} d_{j} \Delta_{f}^{j-2}\right],
$$

and calculate the $[p, q]$ Padé approximant to the expression in square brackets, with $p+q=s-2$. For a given fermion representation $R$, we denote the resultant expression using the $[p, q]$ Padé approximant as $\beta_{\mathrm{IR},[p, q]}^{\prime}$. Note that the $[s-2,0]$ Padé approximant is just the series itself, i.e.,

$$
\beta_{\mathrm{IR},[s-2,0]}^{\prime}=\beta_{\mathrm{IR}, \Delta_{f}^{s}}^{\prime} .
$$

Thus, in particular, $\beta_{\mathrm{IR},[3,0]}^{\prime}=\beta_{\mathrm{IR}, \Delta_{f}^{5}}^{\prime}$. We do not consider this, since we have already obtained evaluations of this series truncation in previous work $[15,16]$.

We focus here on $\mathrm{SU}\left(N_{c}\right)$ theories with fermions in the fundamental, $R=F$ and consider two illustrative values of $N_{c}$, namely 2 and 3 . For $\mathrm{SU}\left(N_{c}\right)$, with $R=F$,

$$
d_{2, F}=\frac{16}{9\left(25 N_{c}^{2}-11\right)} .
$$


This takes the values $d_{2, F}=16 / 801=1.997503 \times 10^{-2}$ for $\mathrm{SU}(2)$ and $d_{2, F}=8 / 963=0.830737 \times 10^{-2}$ for $\mathrm{SU}(3)$.

Although the lowest two nonzero coefficients $d_{2}$ and $d_{3}$ are manifestly positive for any gauge group $G$ and fermion representation $R$, our calculation of $d_{4}$ in [14] and $d_{5}$ in $[15,16]$ showed that these are both negative for $\mathrm{SU}\left(N_{c}\right)$ and $R=F$. Explicitly, for $\mathrm{SU}(2)$ and $\mathrm{SU}(3)$ $[15,16]$,

$$
\begin{aligned}
& \mathrm{SU}(2): \quad \beta_{\mathrm{IR}, F, \Delta_{f}^{5}}^{\prime}=\Delta_{f}^{2}\left[\left(1.99750 \times 10^{-2}+\left(3.66583 \times 10^{-3}\right) \Delta_{f}-\left(3.57303 \times 10^{-4}\right) \Delta_{f}^{2}-\left(2.64908 \times 10^{-5}\right) \Delta_{f}^{3}\right]\right. \\
& \mathrm{SU}(3): \quad \beta_{\mathrm{IR}, F, \Delta_{f}^{5}}^{\prime}=\Delta_{f}^{2}\left[\left(0.83074 \times 10^{-2}\right)+\left(0.98343 \times 10^{-3}\right) \Delta_{f}-\left(0.46342 \times 10^{-4}\right) \Delta_{f}^{2}-\left(0.56435 \times 10^{-5}\right) \Delta_{f}^{3}\right]
\end{aligned}
$$

\section{B. $\mathbf{S U}(2)$}

For SU(2) we calculate the following $[p, q]$ Padé approximants to $\beta_{\mathrm{IR}, F, \Delta_{f}^{5}}^{\prime}$ with $q \neq 0$ :

$$
\begin{aligned}
& \beta_{\mathrm{IR}, F,[2,1]}^{\prime}=\frac{16}{801} \Delta_{f}^{2}\left[\frac{1+0.1093795 \Delta_{f}-0.0314939 \Delta_{f}^{2}}{1-0.0741411 \Delta_{f}}\right] \\
& \beta_{\mathrm{IR}, F,[1,2]}^{\prime}=\frac{16}{801} \Delta_{f}^{2}\left[\frac{1+0.221462 \Delta_{f}}{1+0.0379412 \Delta_{f}+0.0109245 \Delta_{f}^{2}}\right], \\
& \beta_{\mathrm{IR}, F,[0,3]}^{\prime}=\frac{16}{801} \Delta_{f}^{2}\left[\frac{1}{1-0.183521 \Delta_{f}+0.0515673 \Delta_{f}^{2}-0.0114202 \Delta_{f}^{3}}\right] .
\end{aligned}
$$

The [2,1] Padé approximant in (4.6) has a pole at $\Delta_{f}=13.488$, i.e., $N_{f}=-2.388$, which is not relevant. The [1,2] Padé approximant in (4.7) has a complexconjugate pair of poles at $\Delta_{f}=-1.7365 \pm 9.409 i$, of magnitude $\left|\Delta_{f}\right|=9.5675$, considerably larger than $\Delta_{f, \max }=5.449$ and the inferred $\Delta_{f, c r} \simeq 5-6$. Hence, we can use both the [2,1] and [1,2] Padé approximants for our analysis. The $[0,3]$ approximant in $(4.8)$ has a real pole at $\Delta_{f}=4.8906$, i.e., $N_{f}=6.1094$, which lies in the interval $I$ (and also has complex poles at $\Delta_{f}=-0.18158 \pm 4.2272 i$, with magnitude 4.231, smaller than $\Delta_{f, \max }$ and $\Delta_{f, c r}$ ). Consequently, we do not use this [0,3] Padé approximant.
In Table VII we list the values of $\beta_{\mathrm{IR}, F,[2,1]}^{\prime}$ and $\beta_{\mathrm{IR}, F,[1,2]}^{\prime}$, together with the values of $\beta_{\mathrm{IR}, F, \Delta_{f}^{j}}^{\prime}$ with $2 \leq j \leq 5$, as functions of $N_{f}$. As is evident, the values obtained from these Padé approximants are in good agreement with the values obtained from our series expansions in $\Delta_{f}$. For example, for this $\mathrm{SU}(2)$ theory with $N_{f}=8, \beta_{\mathrm{IR}, F,[2,1]}^{\prime}=0.242$ and $\beta_{\mathrm{IR}, F,[1,2]}^{\prime}=0.247$, close to $\beta_{\mathrm{IR}, F, \Delta_{f}^{4}}^{\prime}=0.250$ and $\beta_{\mathrm{IR}, F, \Delta_{f}^{4}}^{\prime}=0.243$.

\section{C. $\mathrm{SU}(3)$}

For $\mathrm{SU}(3)$, we calculate

$$
\begin{aligned}
& \beta_{\mathrm{IR}, F,[2,1]}^{\prime}=\frac{8}{963} \Delta_{f}^{2}\left[\frac{1-0.00339979 \Delta_{f}-0.0199947 \Delta_{f}^{2}}{1-0.121780 \Delta_{f}}\right], \\
& \beta_{\mathrm{IR}, F,[1,2]}^{\prime}=\frac{8}{963} \Delta_{f}^{2}\left[\frac{1+0.117412 \Delta_{f}}{1-\left(0.968004 \times 10^{-3}\right) \Delta_{f}+0.00569298 \Delta_{f}^{2}}\right], \\
& \beta_{\mathrm{IR}, F,[0,3]}^{\prime}=\frac{8}{963} \Delta_{f}^{2}\left[\frac{1}{1-0.118380 \Delta_{f}+0.0195922 \Delta_{f}^{2}-0.00230036 \Delta_{f}^{3}}\right] .
\end{aligned}
$$

The [2,1] Padé approximate in (4.9) has a pole at $\Delta_{f}=8.2115$, i.e., $N_{f}=8.2885$, which lies in the interval $I$. The [1,2] approximant in (4.10) has a complex-conjugate pair of poles at $\Delta_{f}=0.0850 \pm 13.253 i$, with magnitude
13.253, larger than $\Delta_{f, \max }=8.447$ and the inferred $\Delta_{f, c r} \simeq 8$. Finally, the [0,3] approximant in (4.8) has a real pole at $\Delta_{f}=8.488$, i.e., $N_{f}=8.012$, very close to $N_{\ell}=8.05$, and a complex-conjugate pair of poles at 
TABLE VII. Values of $\beta_{\mathrm{IR}, F,[2,1]}^{\prime}$ and $\beta_{\mathrm{IR}, F,[1,2]}^{\prime}$ for $\mathrm{SU}\left(N_{c}\right)$ with $N_{c}=2,3$ and $R=F$ (fundamental) as a function of $N_{f}$. For comparison, we also include the values of $\beta_{\mathrm{IR}, F, \Delta_{f}^{s}}^{\prime}$ from $[14,16]$. The columns list $\beta_{\mathrm{IR}, F, \Delta_{f}^{s}}^{\prime}$ for $2 \leq j \leq 5$, and then $\beta_{\mathrm{IR}, F,[2,1]}^{\prime}$ and $\beta_{\mathrm{IR}, F,[1,2]}^{\prime}$. As discussed in the text, for $\mathrm{SU}(3)$, we use only the $[1,2]$ Padé approximant; this is indicated by the dots $\cdots$ for the entries in the column for $\beta_{\mathrm{IR}, F,[2,1]}^{\prime}$ in the table.

\begin{tabular}{rrlllllll}
\hline \hline$N_{c}$ & $N_{f}$ & $\beta_{\mathrm{IR}, F, \Delta_{f}^{2}}^{\prime}$ & $\beta_{\mathrm{IR}, F, \Delta_{f}^{3}}^{\prime}$ & $\beta_{\mathrm{IR}, F, \Delta_{f}^{4}}^{\prime}$ & $\beta_{\mathrm{IR}, F, \Delta_{f}^{5}}^{\prime}$ & $\beta_{\mathrm{IR}, F,[2,1]}^{\prime}$ & $\beta_{\mathrm{IR}, F,[1,2]}^{\prime}$ \\
\hline 2 & 6 & 0.499 & 0.957 & 0.734 & 0.6515 & 0.603 & 0.719 \\
2 & 7 & 0.320 & 0.554 & 0.463 & 0.436 & 0.424 & 0.454 \\
2 & 8 & 0.180 & 0.279 & 0.250 & 0.243 & 0.242 & 0.247 \\
2 & 9 & 0.0799 & 0.109 & 0.1035 & 0.103 & 0.1025 & 0.103 \\
2 & 10 & 0.0200 & 0.0236 & 0.0233 & 0.0233 & 0.0233 & 0.0233 \\
3 & 9 & 0.467 & 0.882 & 0.7355 & 0.602 & $\cdots$ & 0.669 \\
3 & 10 & 0.351 & 0.621 & 0.538 & 0.473 & $\cdots$ & 0.501 \\
3 & 11 & 0.251 & 0.415 & 0.3725 & 0.344 & $\ldots$ & 0.354 \\
3 & 12 & 0.168 & 0.258 & 0.239 & 0.228 & $\ldots$ & 0.231 \\
3 & 13 & 0.102 & 0.144 & 0.137 & 0.134 & $\ldots$ & 0.135 \\
3 & 14 & 0.0519 & 0.0673 & 0.0655 & 0.0649 & $\cdots$ & 0.0650 \\
3 & 15 & 0.0187 & 0.0220 & 0.0218 & 0.0217 & $\cdots$ & 0.0217 \\
3 & 16 & $2.08 \mathrm{e}-3$ & $2.20 \mathrm{e}-3$ & $2.20 \mathrm{e}-3$ & $2.20 \mathrm{e}-3$ & $\cdots$ & $2.20 \mathrm{e}-3$ \\
\hline \hline
\end{tabular}

$\Delta_{f}=0.0145092 \pm 7.156461 i$, with magnitude 7.156, and hence lying within both the disk $\left|\Delta_{f}\right|<\Delta_{f, \max }$ and the disk $\left|\Delta_{f}\right|<\Delta_{f, c r}$ for this theory. Hence, of these three Padé approximants, we can only use the [1,2] approximant for our study. In Table VII we list the values of $\beta_{\mathrm{IR}, F,[1,2]}^{\prime}$, together with the values of $\beta_{\mathrm{IR}, F, \Delta_{f}^{j}}^{\prime}$ with $2 \leq j \leq 5$, as functions of $N_{f}$. We find that $\beta_{\mathrm{IR}, F,[1,2]}^{\prime}$ is close to the higher-order values of $\beta_{\mathrm{IR}, F, \Delta_{f}^{j}}^{\prime}$; for example, for this $\mathrm{SU}(3)$ theory with $N_{f}=12, \beta_{\mathrm{IR}, F, \Delta_{f}^{5}}^{\prime}=0.228$, while $\beta_{\mathrm{IR}, F,[1,2]}^{\prime}=0.231$.

In [16] we compared our scheme-independent calculations of $\beta_{\mathrm{IR}}^{\prime}$ up to $O\left(\Delta_{f}^{5}\right)$ with a lattice measurement, $\beta_{\mathrm{IR}, F}^{\prime}=$ $0.26(2)$ [53] for this $\mathrm{SU}(3)$ theory with $R=F$ and $N_{f}=12$, finding agreement. Here, we extend this comparison with the new input from our Padé calculation. We recall that the conventional higher-order $n$-loop calculations in powers of $\alpha$ are $\beta_{\mathrm{IR}, 3 \ell, F}^{\prime}=0.2955$ and $\beta_{\mathrm{IR}, 4 \ell, F}^{\prime}=0.282$ [4], which agree with this lattice measurement. As we noted in [16], our higher-order scheme-independent values, namely, $\beta_{\mathrm{IR}, \Delta_{f}^{3}, F}^{\prime}=0.258, \beta_{\mathrm{IR}, \Delta_{f}^{4}, F}^{\prime}=0.239$, and $\beta_{\mathrm{IR}, \Delta_{f}^{5}, F}^{\prime}=0.228$, are also in agreement with this lattice value from [53]. Our new Padé value, $\gamma_{\bar{\psi} \psi, \mathrm{IR}, F,[1,2]}=0.231$, again in reasonable agreement with both our earlier values from our higher-order $n$-loop and scheme-independent series expansions and with the lattice value from [53].

\section{Discussion}

The behavior of $\beta_{\mathrm{IR}}^{\prime}$ in the middle and upper part of the non-Abelian Coulomb phase can be accurately described by the series expansion (2.14), since $\Delta_{f}$ approaches zero as $N_{f}$ approaches $N_{u}$ from below. The behavior of $\beta_{\mathrm{IR}}^{\prime}$ toward the lower end of the NACP is also of considerable interest. As with $\gamma_{\bar{\psi} \psi, \text { IR }}$, one can gain a useful perspective concerning this behavior from known results for a vectorial, asymptotically free $\mathcal{N}=1$ supersymmetric theory with a gauge group $G$ and $N_{f}$ chiral superfields $\Phi_{j}$ and $\tilde{\Phi}_{j}$ transforming according to respective representations $R$ and $\bar{R}$ of $G$ [30-32]. For this supersymmetric gauge theory, the NACP occupies the range (2.32), and $\beta_{\mathrm{IR}}^{\prime} \rightarrow 0$ at the lower end, as well as the upper end, of the NACP [74]. In [17] we calculated Padé approximants for $\beta_{\mathrm{IR}}^{\prime}$ from finite series expansions in $\Delta_{f}$ and found consistency with the vanishing of $\beta_{\mathrm{IR}}^{\prime}$ at the lower end of the NACP (as well as the obvious zero of $\beta_{\mathrm{IR}}^{\prime}$ at the upper end, where $\Delta_{f} \rightarrow 0$ ).

Returning to the nonsupersymmetric theories under consideration here, although $d_{2}$ and $d_{3}$ are manifestly positive for any $G$ and $R$, we found that $d_{4, F}$ and $d_{5, F}$ are negative for $\mathrm{SU}\left(N_{c}\right)$ and $R=F$ [14]. Consequently, as $N_{f}$ decreases below $N_{u}$, i.e., $\Delta_{f}$ increases from zero, $\beta_{\mathrm{IR}}^{\prime}$ is initially positive, and has positive slope. As $N_{f}$ decreases further, i.e., $\Delta_{f}$ increases further, the negative $d_{4, F} \Delta_{f}^{4}+$ $d_{5, F} \Delta_{f}^{5}$ terms become progressively more important. From Table VII, one sees that, for the range of $N_{f}$ included, $\beta_{\mathrm{IR}, F, \Delta_{f}^{5}}^{\prime}$ and the resultant Padé approximants are continuing to increase with decreasing $N_{f}$. Evidently, the negative $d_{4, F} \Delta_{f}^{4}+d_{5, F} \Delta_{f}^{5}$ terms are not sufficiently large in magnitude to cause $\beta_{\mathrm{IR}}^{\prime}$ to vanish at the lower end of the NACP. Hence, one needs to calculate the scheme-independent series expansion for $\beta_{\mathrm{IR}}^{\prime}$, Eq. (2.14), to higher order in $\Delta_{f}$ to see this turnover. Insofar as the behavior of $\beta_{\mathrm{IR}}^{\prime}$ in the $\mathcal{N}=1$ supersymmetric gauge theory is at least a qualitative guide to the nonsupersymmetric gauge theory, then it suggests that the exact $\beta_{\mathrm{IR}}^{\prime}$ would reach a maximum in the NACP and then would decrease and vanish as $N_{f}$ decreased to the lower end of this NACP. We suggest that this is a plausible behavior for the nonsupersymmetric theory.

\section{E. LNN limit}

The coefficients $\hat{d}_{j, F}$ with $2 \leq j \leq 4$ were given in [14], and $\hat{d}_{5, F}$ was given in [16]. For reference, these are

$\hat{d}_{2, F}=\frac{2^{4}}{3^{2} \cdot 5^{2}}=0.0711111$,
$\hat{d}_{3, F}=\frac{416}{3^{3} \cdot 5^{4}}=2.465185 \times 10^{-2}$,

$\hat{d}_{4, F}=\frac{5868512}{3^{5} \cdot 5^{10}}-\frac{5632}{3^{4} \cdot 5^{6}} \zeta_{3}=-\left(2.876137 \times 10^{-3}\right)$,

and 


$$
\hat{d}_{5, F}=-\frac{9542225632}{3^{6} \cdot 5^{14}}-\frac{1444864}{3^{5} \cdot 5^{9}} \zeta_{3}+\frac{360448}{3^{5} \cdot 5^{8}} \zeta_{5}=-\left(1.866490 \times 10^{-3}\right) .
$$

Analogously to Eq. (4.1), we write

$$
\beta_{\mathrm{IR}, \Delta_{r}^{s}}^{\prime}=\sum_{j=2}^{s} \hat{d}_{j, F} \Delta_{r}^{j}=\hat{d}_{2, F} \Delta_{r}^{2}\left[1+\frac{1}{\hat{d}_{2, F}} \sum_{j=3}^{s} \hat{d}_{j, F} \Delta_{r}^{j-2}\right],
$$

and calculate the $[p, q]$ Padé approximant to the expression in square brackets, with $p+q=s-2$. We have calculated analytic results for Padé approximants to $\gamma_{\bar{\psi} \mu, \mathrm{IR}, F, \Delta_{r}^{5}}$. As before, we present these in numerical form. We find

$$
\begin{aligned}
& \beta_{\mathrm{IR}, F, L N N,[2,1]}^{\prime}=\frac{16}{225} \Delta_{r}^{2}\left[\frac{1-0.302290 \Delta_{r}-0.265417 \Delta_{r}^{2}}{1-0.648957 \Delta_{r}}\right], \\
& \beta_{\mathrm{IR}, F, L N N,[1,2]}^{\prime}=\frac{16}{225} \Delta_{r}^{2}\left[\frac{1+0.270549 \Delta_{r}}{1-\left(0.0761180 \Delta_{r}+0.0668333 \Delta_{r}^{2}\right.}\right], \\
& \beta_{\mathrm{IR}, F, L N N,[0,3]}^{\prime}=\frac{16}{225} \Delta_{r}^{2}\left[\frac{1}{1-0.346667 \Delta_{r}+0.160623 \Delta_{r}^{2}-0.0434565 \Delta_{r}^{3}}\right] .
\end{aligned}
$$

The [2,1] Padé approximant has a pole at $\Delta_{r}=1.54093$, i.e., $r=3.95907$. This is in the interval $I$ and the inferred NACP, so we cannot use this approximant for our work. The [1,2] Padé approximant has a complex-conjugate pair of poles at $\Delta_{r}=0.56946 \pm 3.82601 i$ with magnitude $\left|\Delta_{r}\right|=3.86815$, which is greater than both $\Delta_{r, \max }=$ 2.8846 and $\Delta_{c r}=2.6$. Hence, we can use this approximant. Finally, the $[0,3]$ Padé approximant has a pole at $\Delta_{r}=3.36019$, i.e., $r=2.13981$ and a complex-conjugate pair of poles at $\Delta_{r}=0.168003 \pm 2.611526 i$ with magnitude 2.6169. Although the real pole is outside of the interval $I$ and the inferred NACP, the complex poles lie within the disks $\left|\Delta_{r}\right|<\Delta_{r, \max }$ and $\left|\Delta_{r}\right|<\Delta_{c r}$, so we do not use this $[0,3]$ approximant. In Table VIII we list the values of $\beta_{\mathrm{IR}, F, L N N,[1,2]}^{\prime}$ as a function of $r$, together with our previously calculated $\beta_{\mathrm{IR}, F, \Delta_{r}^{s}}^{\prime}$ with $2 \leq s \leq 5$ for comparison. We find that the values of the [1,2] Padé approximant are close to those of the high-order $\beta_{\mathrm{IR}, F, \Delta_{r}^{s}}^{\prime}$; for example, at $r=4.0, \beta_{\mathrm{IR}, F, \Delta_{r}^{5}}^{\prime}=0.214$ while $\beta_{\mathrm{IR}, F, L N N,[1,2]}^{\prime}=0.217$. As $r$ decreases toward the lower end of the non-Abelian Coulomb phase, $\beta_{\mathrm{IR}, F, L N N,[1,2]}^{\prime}$ becomes slightly larger than $\beta_{\mathrm{IR}, F, \Delta_{r}^{5}}^{\prime}$, just as was the case as $N_{f}$ decreased toward the

\begin{tabular}{|c|c|c|c|c|c|}
\hline$r$ & $\beta_{\mathrm{IR}, F, L N N, \Delta_{r}^{2}}^{\prime}$ & $\beta_{\mathrm{IR}, F, L N N, \Delta_{r}^{3}}^{\prime}$ & $\beta_{\mathrm{IR}, F, L N N, \Delta_{r}^{4}}^{\prime}$ & $\beta_{\mathrm{IR}, F, L N N, \Delta_{r}^{5}}^{\prime}$ & $\beta_{\mathrm{IR}, F, L N N,[1,2]}^{\prime}$ \\
\hline 2.8 & 0.518 & 1.004 & 0.851 & 0.583 & 0.700 \\
\hline 3.0 & 0.444 & 0.830 & 0.717 & 0.535 & 0.607 \\
\hline 3.2 & 0.376 & 0.676 & 0.596 & 0.475 & 0.518 \\
\hline 3.4 & 0.314 & 0.542 & 0.486 & 0.410 & 0.433 \\
\hline 3.6 & 0.257 & 0.426 & 0.388 & 0.342 & 0.354 \\
\hline 3.8 & 0.2055 & 0.327 & 0.303 & 0.276 & 0.282 \\
\hline 4.0 & 0.160 & 0.243 & 0.229 & 0.214 & 0.217 \\
\hline 4.2 & 0.120 & 0.174 & 0.166 & 0.159 & 0.160 \\
\hline 4.4 & 0.0860 & 0.119 & 0.115 & 0.112 & 0.112 \\
\hline 4.6 & 0.0576 & 0.0756 & 0.0737 & 0.0726 & 0.0727 \\
\hline 4.8 & 0.0348 & 0.0433 & 0.0426 & 0.0423 & 0.0423 \\
\hline 5.0 & 0.0178 & 0.0209 & 0.0207 & 0.0206 & 0.0206 \\
\hline 5.2 & $0.640 \mathrm{e}-2$ & $0.707 \mathrm{e}-2$ & $0.704 \mathrm{e}-2$ & $0.704 \mathrm{e}-2$ & $0.704 \mathrm{e}-2$ \\
\hline 5.4 & $0.711 \mathrm{e}-3$ & $0.736 \mathrm{e}-3$ & $0.735 e-3$ & $0.735 e-3$ & $0.735 e-3$ \\
\hline
\end{tabular}
respective lower ends of the NACP in the specific $\mathrm{SU}(2)$ and $\mathrm{SU}(3)$ theories discussed above.

TABLE VIII. Values of $\beta_{\mathrm{IR}, F, L N N,[1,2]}^{\prime}$ in the LNN limit, as a function of the ratio $r$ defined in Eq. (2.15). For comparison, we also include the values of $\beta_{\mathrm{IR}, F, L N N, \Delta_{r}^{s}}^{\prime}$ from $[14,16]$ for $2 \leq s \leq 5$. 
TABLE IX. Values of higher-order $\hat{d}_{j, F, L N N,[1,2]}$ obtained by Taylor series expansions of $\beta_{\mathrm{IR}, F, L N N,[1,2]}^{\prime}$ in the LNN limit. The notation $a \mathrm{e}-\mathrm{n}$ means $a \times 10^{-n}$.

\begin{tabular}{cc}
\hline \hline$j$ & $\hat{d}_{j, F, L N N,[1,2]}$ \\
\hline 6 & $0.5014815 \mathrm{e}-4$ \\
7 & $1.285608 \mathrm{e}-4$ \\
8 & $0.643423 \mathrm{e}-5$ \\
9 & $-0.81023751 \mathrm{e}-5$ \\
10 & $-1.046757 \mathrm{e}-6$ \\
11 & $0.461831 \mathrm{e}-6$ \\
12 & $1.051119 \mathrm{e}-7$ \\
\hline \hline
\end{tabular}

As with $\gamma_{\bar{\psi} \psi, I R, F, L N N}$, it is of interest to carry out a Taylor series expansion of $\beta_{\mathrm{IR}, F, L N N,[1,2]}^{\prime}$ to determine its prediction for the coefficients $\hat{d}_{j, F}$ with $j \geq 6$. We have done this and present the resultant $\hat{d}_{j, F}$ coefficients in Table IX.

\section{CONCLUSIONS}

In this paper, we have presented several new results on the anomalous dimension, $\gamma_{\bar{\psi} \psi, \text { IR }}$, and the derivative of the beta function, $\beta_{\mathrm{IR}}^{\prime}$, at an infrared fixed point of the renormalization group in vectorial, asymptotically free $\mathrm{SU}\left(N_{c}\right)$ gauge theories with $N_{f}$ fermions transforming according to several representations $R$, including the fundamental, adjoint, and rank-2 symmetric tensor. We have used our series for $\gamma_{\bar{\psi} \psi \text {,IR }}$ to $O\left(\Delta_{f}^{4}\right)$ to calculate Padé approximants and have evaluated these to obtain further estimates of $\gamma_{\bar{\psi} \psi, \text { IR }}$. Our new results using these Padé approximants are consistent with our earlier results using the series themselves calculated to $O\left(\Delta_{f}^{4}\right)$. We have compared the values of $\gamma_{\bar{\psi} \psi \text {,IR }}$ with lattice measurements for various theories. Taylor-series expansions of the Padé approximants have been calculated to determine their predictions for higher-order coefficients. We have found that all of the Padé approximants that we have calculated that satisfy the requisite constraints (absence of poles in the disks $\left|\Delta_{f}\right|<\Delta_{f, \max }$ and $\left|\Delta_{f}\right|<\Delta_{f, c r}$ in the complex $\Delta_{f}$ plane) yield Taylor-series expansions with positive coefficients $\kappa_{j}$, providing further support for our earlier conjecture that the $\kappa_{j}$ are positive. We have also used our Padé results to obtain new estimates of the value of $N_{f, c r}$ at the lower end of the non-Abelian Coulomb phase for various $N_{c}$ and $R$. Since, for a given $\mathrm{SU}\left(N_{c}\right)$ gauge group and fermion representation $R$, the upper end of the NACP, namely $N_{u}$, is known exactly, these estimates of $N_{f, c r}$ are equivalently estimates of the extent of the nonAbelian Coulomb phase, as a function of $N_{f}$, for each of the theories that we have considered. In a different but related application, our values of $N_{f, c r}$ are useful for the phenomenological program of constructing and studying quasiconformal gauge theories to explore ideas for possible ultraviolet completions of the Standard Model. This is because, for a given gauge group $G$ and fermion representation $R$, one must choose $N_{f}$ to be slightly below $N_{f, c r}$ (requiring that one know $N_{f, c r}$ ) in order to achieve the quasiconformal behavior whose spontaneous breaking via formation of fermion condensates could have the potential to yield a light, dilatonic Higgs-like scalar. We have carried out calculations of Padé approximants for $\beta_{\mathrm{IR}}^{\prime}$, using our series to $O\left(\Delta_{f}^{5}\right)$ for these theories. Again, the results for $\beta_{\mathrm{IR}}^{\prime}$ obtained from these Padé approximants are consistent with, and extend, our earlier analyses using the series themselves. Our values for $\gamma_{\bar{\psi} \psi, \text { IR }}$ and $\beta_{\mathrm{IR}}^{\prime}$ obtained with Padé approximants provide further information about fundamental properties of conformal field theories. Finally, we have presented new analytic and numerical results assessing the accuracy of a series expansion of $\gamma_{\bar{\psi} \psi, \text { IR }}$ to finite order in powers of $\Delta_{f}$ by comparison with the exactly known expression in an $\mathcal{N}=1$ supersymmetric gauge theory, showing that an expansion to $O\left(\Delta_{f}^{4}\right)$ is quite accurate throughout the entire non-Abelian Coulomb phase of this supersymmetric theory.

\section{ACKNOWLEDGMENTS}

The research of T. A. R. and R. S. was supported in part by the Danish National Research Foundation Grant No. DNRF90 to CP3-Origins at SDU and by the U.S. National Science Foundation Grant No. NSF-PHY-161620628 , respectively.
[1] Some early analyses of relations between scale and conformal invariance are A. Salam, Ann. Phys. (N.Y.) 53, 174 (1969); A. M. Polyakov, JETP Lett. 12, 381 (1970); D. J. Gross and J. Wess, Phys. Rev. D 2, 753 (1970); C. G. Callan, S. Coleman, and R. Jackiw, Ann. Phys. (N.Y.) 59, 42 (1970); More recent papers include J. Polchinski, Nucl. Phys. B303, 226 (1988); S. El-Showk, Y. Nakayama, and
S. Rychkov, Nucl. Phys. B848, 578 (2011); J.-F. Fortin, B. Grinstein, and A. Stergiou, J. High Energy Phys. 01 (2013) 184; Y. Nakayama, Phys. Rep. 569, 1 (2015); A. Dymarsky, Z. Komargodski, A. Schwimmer, and S. Thiessen, J. High Energy Phys. 10 (2015) 171.

[2] T. A. Ryttov and R. Shrock, Phys. Rev. D 83, 056011 (2011). 
[3] C. Pica and F. Sannino, Phys. Rev. D 83, 035013 (2011).

[4] R. Shrock, Phys. Rev. D 87, 105005 (2013); 87, 116007 (2013).

[5] T. A. Ryttov and R. Shrock, Phys. Rev. D 94, 105015 (2016).

[6] The assumption of massless fermions incurs no loss of generality, since if a fermion had a nonzero mass $m_{0}$, it would be integrated out of the effective field theory at scales $\mu<m_{0}$, and hence would not affect the IR limit $\mu \rightarrow 0$ considered here.

[7] T. Banks and A. Zaks, Nucl. Phys. B196, 189 (1982).

[8] G. Grunberg, Phys. Rev. D 46, 2228 (1992); E. Gardi and M. Karliner, Nucl. Phys. B529, 383 (1998); E. Gardi and G. Grunberg, J. High Energy Phys. 03 (1999) 024.

[9] D. J. Gross, in Methods in Field Theory, Les Houches 1975, edited by R. Balian and J. Zinn-Justin (North Holland, Amsterdam, 1976), p. 141.

[10] S. L. Adler, J. C. Collins, and A. Duncan, Phys. Rev. D 15, 1712 (1977); J. C. Collins, A. Duncan, and S. Joglekar, Phys. Rev. D 16, 438 (1977); N. K. Nielsen, Nucl. Phys. B120, 212 (1977); see also H. Kluberg-Stern and J.-B. Zuber, Phys. Rev. D 12, 467 (1975).

[11] S. S. Gubser, A. Nellore, S. S. Pufu, and E. D. Rocha, Phys. Rev. Lett. 101, 131601 (2008).

[12] T. A. Ryttov, Phys. Rev. Lett. 117, 071601 (2016).

[13] T. A. Ryttov and R. Shrock, Phys. Rev. D 94, 105014 (2016).

[14] T. A. Ryttov and R. Shrock, Phys. Rev. D 94, 125005 (2016).

[15] T. A. Ryttov and R. Shrock, Phys. Rev. D 95, 085012 (2017).

[16] T. A. Ryttov and R. Shrock, Phys. Rev. D 95, 105004 (2017).

[17] T. A. Ryttov and R. Shrock, Phys. Rev. D 96, 105018 (2017).

[18] T. A. Ryttov and R. Shrock, Phys. Rev. D 96, 105015 (2017).

[19] For discussions of other schemes, see, e. g., T. A. Ryttov and R. Shrock, Phys. Rev. D 86, 065032 (2012); T. A. Ryttov and R. Shrock, Phys. Rev. D 86, 085005 (2012); R. Shrock, Phys. Rev. D 88, 036003 (2013); T. A. Ryttov, Phys. Rev. D 90, 056007 (2014); and J. A. Gracey and R. M. Simms, Phys. Rev. D 91, 085037 (2015).

[20] Here and elsewhere, when an expression is given for $N_{f}$ that formally evaluates to a nonintegral real value, it is understood implicitly that one infers an appropriate integral value from it.

[21] Let $T_{R}^{a}$ denote the generators of the Lie algebra of a group $G$ in the representation $R$, where $a$ is a group index, and let $d_{R}$ denote the dimension of $R$. The Casimir invariants $C_{2}(R)$ and $T_{R}$ are defined as follows: $T_{R}^{a} T_{R}^{a}=C_{2}(R) I$, where here $I$ is the $d_{R} \times d_{R}$ identity matrix, and $\operatorname{Tr}_{R}\left(T_{R}^{a} T_{R}^{b}\right)=T(R) \delta^{a b}$. For a fermion $f$ transforming according to a representation $R$, we often use the equivalent compact notation $T_{f} \equiv T(R)$ and $C_{f} \equiv C_{2}(R)$. We also use the notation $C_{A} \equiv C_{2}(A) \equiv C_{2}(G)$.

[22] In some early work there was also an attempt to apply the $\Delta_{f}$ expansion to actual quantum chromodynamics (QCD); however, the properties of an asymptotically free gauge theory are qualitatively different for $\Delta_{f}<\Delta_{f, c r}$ in the (chirally symmetric, deconfined) non-Abelian Coulomb phase and for $\Delta_{f}>\Delta_{f, c r}$ in QCD, with its confinement and spontaneous chiral symmetry breaking.

[23] Lattice simulations of vectorial gauge theories have made progress toward the determination of $N_{f, c r}$ for several gauge groups $G$ and fermion representations $R$; recent reviews include Lattice-2016 at https://www.southampton.ac.uk/ lattice2016; T. DeGrand, Rev. Mod. Phys. 88, 015001 (2016); Lattice for BSM 2017 Workshop at http://wwwhep.colorado.edu/ eneil/lbsm17; and Lattice-2017 at http:// wpd.ugr.es/ lattice2017.

[24] G. Mack, Commun. Math. Phys. 55, 1 (1977); B. Grinstein, K. Intriligator, and I. Rothstein, Phys. Lett. B 662, 367 (2008); Y. Nakayama, Phys. Rep. 569, 1 (2015), op. cit., [1]. See also R. Rattazzi, V. S. Rychkov, E. Tonni, and A. Vichi, J. High Energy Phys. 12 (2008) 031; H. Iha, H. Makino, and H. Suzuki, Prog. Theor. Exp. Phys. 2016, 053 B03 (2016).

[25] For a given $G$ and $R$, if $N_{f}<N_{f, c r}$, so that there is spontaneous chiral symmetry breaking, then even if the perturbative beta function with the original $N_{f}$ fermions exhibits an IR zero at some value $\alpha_{\mathrm{IR}}$, this is only approximate, since the theory flows away from this value as the fermions gain dynamical mass and are integrated out, leaving a pure gluonic low-energy effective field theory with a different beta function.

[26] J. A. Gracey, Phys. Lett. B 488, 175 (2000).

[27] F. Herzog, B. Ruijl, T. Ueda, J. A. M. Vermaseren, and A. Vogt, J. High Energy Phys. 02 (2017) 090.

[28] K. G. Chetyrkin, Phys. Lett. B 404, 161 (1997); J. A. M. Vermaseren, S. A. Larin, and T. van Ritbergen, Phys. Lett. B 405, 327 (1997).

[29] Parenthetically, we note that a different approach to estimate $N_{f, c r}$ and associated spontaneous chiral symmetry breaking $(\mathrm{S} \chi \mathrm{SB})$ relies upon approximate solutions, in the ladder approximation, of the Schwinger-Dyson equation for the fermion propagator. This leads to $\mathrm{S} \chi \mathrm{SB}$ at $\gamma_{\bar{\psi} \psi} \simeq 1$; see K. Yamawaki, M. Bando, and K. Matumoto, Phys. Rev. Lett. 56, 1335 (1986); T. Appelquist, D. Karabali, and L. C. R. Wijewardhana, Phys. Rev. Lett. 57, 957 (1986). In contrast, the rigorous upper bound (2.12) allows $\gamma_{\bar{\psi} \psi \psi}$ to lie above 1 in the (chirally symmetric) non-Abelian Coulomb phase, as discussed in Ref. [24].

[30] V. A. Novikov, M. A. Shifman, A. I. Vainshtein, and V. I. Zakharov (NSVZ), Phys. Lett. B 166, 329 (1986).

[31] N. Seiberg, Nucl. Phys. B435, 129 (1995).

[32] K. A. Intriligator and N. Seiberg, Nucl. Phys. B, Proc. Suppl. 45, 1 (1996); M. A. Shifman, Prog. Part. Nucl. Phys. 39, 1 (1997).

[33] T. A. Ryttov and R. Shrock, Phys. Rev. D 85, 076009 (2012).

[34] We note that, although $\gamma_{M \text {,IR }}$ saturates its conformality upper bound at the lower end of the NACP in this $\mathcal{N}=1$ supersymmetric gauge theory, anomalous dimensions of various other composite chiral superfield operator products were calculated in [17], and it was found that they do not saturate their conformality upper bounds.

[35] T. A. Ryttov and F. Sannino, Phys. Rev. D 76, 105004 (2007).

[36] G. A. Baker and P. Graves-Morris, Padé Approximants, Encyclopedia of Math. (Addison-Wesley, Reading, MA, 1981), Vol. 13. 
[37] For reviews, see, e. g., J. Zinn-Justin, Quantum Field Theory and Critical Phenomena, 4th ed. (Oxford University Press, Oxford, England, 2002) and H. Kleinert and V. SchulteFrohlinde, Critical Properties of $\phi^{4}$ Theories (World Scientific, Singapore, 2001).

[38] J. L. Basdevant, D. Bessis, and J. Zinn-Justin, Phys. Lett. 27B, 230 (1968); J. L. Basdevant and B. W. Lee, Nucl. Phys. B13, 182 (1969).

[39] Our notation follows I-H. Lee and R. E. Shrock, Phys. Rev. B 36, 3712 (1987); J. Phys. A 21, 3139 (1988); V. Matveev and R. Shrock, J. Phys. A 28, 1557 (1995).

[40] M. A. Samuel, J. Ellis, and M. Karliner, Phys. Rev. Lett. 74, 4380 (1995); Phys. Lett. B 400, 176 (1997).

[41] F. A. Chishtie, V. Elias, and T. G. Steele, Phys. Lett. B 446, 267 (1999).

[42] R. Shrock, Phys. Rev. D 90, 065023 (2014); 94, 125026 (2016); 96, 056010 (2017).

[43] G. Choi, T. A. Ryttov, and R. Shrock, Phys. Rev. D 95, 025012 (2017).

[44] V. Leino, J. Rantaharju, T. Rantalaiho, K. Rummukainen, J. M. Suorsa, and K. Tuominen, Phys. Rev. D 95, 114516 (2017); See also H. Ohki et al., Proc. Sci., LATTICE2010 (2010) 066 [arXiv:1011.0373]; C. Y.-H. Huang et al., Proc. Sci., LATTICE2015 (2016) 224 [arXiv:1511.01968].

[45] SU(2) gauge theories with $R=F$ and $N_{f}=8$ have been of past interest in the context of ideas for ultraviolet completions of the Standard Model; see T. Appelquist and R. Shrock, Phys. Lett. B 548, 204 (2002); Phys. Rev. Lett. 90, 201801 (2003); T. Appelquist, M. Piai, and R. Shrock, Phys. Rev. D 69, 015002 (2004); N. C. Christensen and R. Shrock, Phys. Rev. Lett. 94, 241801 (2005) and references therein.

[46] V. Leino, K. Rummukainen, J. Suorsa, K. Tuominen, and S.Tahatinen, arXiv:1707.04722.

[47] F. Bursa, L. Del Debbio, L. Keegan, C. Pica, and T. Pickup, Phys. Lett. B 696, 374 (2011); T. Karavirta, J. Rantaharju, K. Rummukainen, and K. Tuominen, J. High Energy Phys. 05 (2012) 003; M. Hayakawa, K. I. Ishikawa, S. Takeda, M. Tomii, and N. Yamada, Phys. Rev. D 88, 094506 (2013); T. Appelquist et al. (LSD Collaboration), Phys. Rev. Lett. 112, 111601 (2014).

[48] T. Appelquist et al. (LSD Collaboration), Phys. Rev. D 84, 054501 (2011).

[49] T. DeGrand, Phys. Rev. D 84, 116901 (2011).

[50] Y. Aoki et al. (LatKMI Collaboration), Phys. Rev. D 86, 054506 (2012).

[51] A. Hasenfratz, A. Cheng, G. Petropoulos, and D. Schaich, Proc. Sci., LATTICE2012 (2012) 034 [arXiv:1207.7162].

[52] A. Hasenfratz, A. Cheng, G. Petropoulos, and D. Schaich, Proc. Sci., LATTICE2013 (2014) 075 [arXiv:1310.112].

[53] A. Hasenfratz and D. Schaich, arXiv:1610.10004.

[54] M. P. Lombardo, K. Miura, T. J. Nunes da Silva, and E. Pallante, J. High Energy Phys. 12 (2014) 183; See also A. Deuzeman, M. P. Lombardo, T. Nunes Da Silva, and
E. Pallante, Phys. Lett. B 720, 358 (2013); K. Miura, M. P. Lombardo, and E. Pallante, Phys. Lett. B 710, 676 (2012).

[55] Z. Fodor, K. Holland, J. Kuti, D. Nogradi, C. Schroeder, and C. H. Wong, Proc. Sci., LATTICE2012 (2012) 279 [arXiv: 1211.4238]; Z. Fodor, K. Holland, J. Kuti, S. Mondal, D. Nogradi, and C. H. Wong, Phys. Rev. D 94, 091501 (2016). These authors argue that the $\mathrm{SU}(3), R=F, N_{f}=12$ theory is chirally broken, so the quoted $\gamma_{\bar{\psi} \psi, \text { IR, } F}$ is an effective value.

[56] A. Hasenfratz, C. Rebbi, and O. Witzel, arXiv:1708.03385.

[57] T. Appelquist et al. (LSD Collaboration), arXiv:1204.6000.

[58] Y. Aoki et al. (LatKMI Collaboration), Phys. Rev. D 87, 094511 (2013); 89, 111502 (2014); 96, 014508 (2017).

[59] T. Appelquist et al. (LSD Collaboration), Phys. Rev. D 90, 114502 (2014); 93, 114514 (2016) and references therein.

[60] T. Appelquist, J. Ingoldby, and M. Piai, J. High Energy Phys. 07 (2017) 035.

[61] M. Hansen, K. Langæble, and F. Sannino, Phys. Rev. D 95, 036005 (2017).

[62] D. D. Dietrich and F. Sannino, Phys. Rev. D 75, 085018 (2007); S. Catterall and F. Sannino, Phys. Rev. D 76, 034504 (2007).

[63] For relevant discussions of effects of adding four-fermion operators on these types of theories, see, e.g., K. Yamawaki, arXiv:hep-ph/9603293; M. Kurachi, K. Yamawaki, and R. Shrock, Phys. Rev. D 76, 035003 (2007); H. S. Fukano and F. Sannino, Phys. Rev. D 82, 035021 (2010), and references therein.

[64] S. Catterall, L. Del Debbio, J. Giedt, and L. Keegan, Proc. Sci., LATTICE2010 (2010) 057 [arXiv:1010.5909].

[65] L. Del Debbio, B. Lucini, A. Patella, C. Pica, and A. Rago, Phys. Rev. D 82, 014510 (2010). See also A. Patella, Phys. Rev. D 86, 025006 (2012), which reported $\gamma_{\bar{\psi} \mu, I R, A}=$ $0.371(20)$ for this $\mathrm{SU}(2), N_{f}=2$ theory.

[66] T. DeGrand, Y. Shamir, and B. Svetitsky, Phys. Rev. D 83, 074507 (2011).

[67] T. Appelquist et al. (LSD Collaboration), Phys. Rev. D 84, 054501 (2011).

[68] J. Giedt and E. Weinberg, Phys. Rev. D 85, 097503 (2012); J. Giedt, Int. J. Mod. Phys. A 31, 1630011 (2016).

[69] J. Rantaharju, T. Rantalaiho, K. Rummukainen, and K. Tuominen, Phys. Rev. D 93, 094509 (2016).

[70] G. Bergner, P. Giudice, I. Montvay, and S. Piemonte, Phys. Rev. D 96, 034504 (2017).

[71] T. DeGrand, Y. Shamir, and B. Svetitsky, Phys. Rev. D 87, 074507 (2013).

[72] Z. Fodor, K. Holland, J. Kuti, D. Nogradi, C. Schroeder, and C. H. Wong, Phys. Lett. B 718, 657 (2012).

[73] T. Nunes da Silva, E. Pallante, and L. Robroek, arXiv: 1609.06298.

[74] D. Anselmi, M. T. Grisaru, and A. A. Johansen, Nucl. Phys. B491, 221 (1997). 